\title{
Endocrine remodelling of the adult intestine sustains reproduction in Drosophila
}

\section{Tobias Reiff $^{1 \dagger}$, Jake Jacobson ${ }^{2 \dagger}$, Paola Cognigni ${ }^{2+\ddagger}$, Zeus Antonello ${ }^{1 \dagger}$, Esther Ballesta ${ }^{1}$, Kah Junn Tan ${ }^{3}$, Joanne Y Yew ${ }^{3,48}$, Maria Dominguez ${ }^{1 *}$, Irene Miguel-Aliaga ${ }^{2 *}$}

${ }^{1}$ Instituto de Neurociencias, Consejo Superior de Investigaciones Cientificas, Universidad Miguel Hernández, Alicante, Spain; ${ }^{2}$ MRC Clinical Sciences Centre, Imperial College London, London, United Kingdom; ${ }^{3}$ Temasek Life Sciences Laboratory, Singapore, Singapore; ${ }^{4}$ Department of Biological Sciences, National University of Singapore, Singapore, Singapore
${ }^{\star}$ For correspondence: m.dominguez@umh.es (MD); i.miguel-aliaga@imperial.ac. uk (IM)

tThese authors contributed equally to this work

Present address: ${ }^{\ddagger}$ Centre for Neural Circuits and Behaviour, University of Oxford, Oxford, United Kingdom; ${ }^{\circledR}$ Pacific Biosciences Research Center, University of Hawai'i at Mānoa, Honolulu, United States

Competing interests: The authors declare that no competing interests exist.

Funding: See page 16

Received: 09 February 2015

Accepted: 06 June 2015

Published: 28 July 2015

Reviewing editor: Matthew Freeman, University of Oxford, United Kingdom

Copyright Reiff et al. This article is distributed under the terms of the Creative Commons Attribution License, which permits unrestricted use and redistribution provided that the original author and source are credited.
Abstract The production of offspring is energetically costly and relies on incompletely understood mechanisms that generate a positive energy balance. In mothers of many species, changes in key energy-associated internal organs are common yet poorly characterised functionally and mechanistically. In this study, we show that, in adult Drosophila females, the midgut is dramatically remodelled to enhance reproductive output. In contrast to extant models, organ remodelling does not occur in response to increased nutrient intake and/or offspring demands, but rather precedes them. With spatially and temporally directed manipulations, we identify juvenile hormone $(\mathrm{JH})$ as an anticipatory endocrine signal released after mating. Acting through intestinal bHLH-PAS domain proteins Methoprene-tolerant (Met) and Germ cell-expressed (Gce), JH signals directly to intestinal progenitors to yield a larger organ, and adjusts gene expression and sterol regulatory element-binding protein (SREBP) activity in enterocytes to support increased lipid metabolism. Our findings identify a metabolically significant paradigm of adult somatic organ remodelling linking hormonal signals, epithelial plasticity, and reproductive output.

DOI: 10.7554/eLife.06930.001

\section{Introduction}

Reproduction is energetically costly. Mothers can adjust their energy balance to maximise their reproductive success through well-established neural mechanisms that match food intake to their enhanced energy requirements (Roa and Tena-Sempere, 2014). However, less wellunderstood changes also occur in many animals during reproduction; internal organs, such as the liver, pancreas, and gastrointestinal tract, increase their size and adapt their physiology, potentially contributing to an increased generation and delivery of nutrients (Hammond, 1997; Speakman, 2008).

Establishment of a positive energy balance may be particularly important to animals with a reproductive strategy that involves rapid production of large numbers of progeny. Drosophila melanogaster females can lay up to 100 eggs per day at the peak of their fertility in early life (David et al., 1974; Klepsatel et al., 2013). We hypothesised that such demands may rely on major regulatory responses, which are amenable to genetic investigation in this model system. A network of organs and tissues in Drosophila perform many of the same basic functions as those found in mammals (Padmanabha and Baker, 2014), so we sought to explore the nature and significance of organ plasticity during reproduction. 
eLife digest Producing offspring places extra energy demands on individuals. Female animals-which generally invest more time and resources than the males-need to ensure that sufficient nutrients reach their offspring during pregnancy and lactation. The small intestines of many female animals increase in size during this period, but it is not clear to what extent these changes help to maximise reproduction, or how they are regulated.

Reiff, Jacobson, Cognigni, Antonello et al. investigated what happens to the middle section of the gut in female fruit flies after mating. A fly's 'midgut' performs a similar role to the small intestine in humans and other mammals. The experiments show that mating increases the numbers of cells in the midgut so that it increases in size.

These changes are due to a hormone called 'juvenile hormone', which is released after the fly mates. In particular cells of the midgut, juvenile hormone also regulates some genes involved in the metabolism of lipids. If the activity of juvenile hormone is blocked in these cells, the female flies produce fewer eggs. These changes in the midgut still happen in mutant flies that cannot produce eggs and don't increase their food intake after they mate. Therefore, the changes appear to prepare flies for the increased nutritional demands rather than being caused by it.

Altogether, these findings reveal that changes in the midgut play an important role in the ability of female fruit flies to reproduce. Similar changes to the gut may also increase reproductive success in humans and other mammals. However, if the changes are maintained after reproduction, it is possible that they may contribute to weight gain and an increased risk of cancer in females after pregnancy.

DOI: 10.7554/eLife.06930.002

\section{Results}

\section{The adult midgut is remodelled in female flies after mating}

Female flies undergo multiple post-mating adaptations including changes in digestive physiology (Cognigni et al., 2011). This prompted us to characterise possible intestinal changes occurring during the phase of peak fertility (David et al., 1974; Klepsatel et al., 2013). We focused on the midgut epithelium because of its major digestive/absorptive roles (Lemaitre and Miguel-Aliaga, 2013). In the midgut epithelium, long-lived progenitors (intestinal stem cells (ISCs)) divide to self-renew and to give rise to committed progenitors (called enteroblasts (EBs)), which directly differentiate into two types of progeny: absorptive enterocytes (ECs) and enteroendocrine cells (EECs) (Jiang and Edgar, 2012). We found that mating increases the number of both dividing and differentiating midgut cells, as revealed by phospho-Histone $\mathrm{H} 3(\mathrm{pH} 3)$ stainings and temporal analyses of progenitors and their descendants using the dual-labelling system escargot-Repressible Dual Differential Marker (esgReDDM, Antonello et al., 2015) (Figure 1A,C,E). The midgut of mated females also becomes visibly larger; gut diameter measurements were suggestive of a net increase in the number of postmitotic intestinal cells (Figure 1B,D, Figure 1-figure supplement 1): an increase that we confirmed by cell number and density counts (Figure 1F, Figure 1-figure supplement 1). Concurrent with midgut re-sizing, we observed mating-dependent activation of the single Drosophila homologue of the mammalian family of sterol regulatory element-binding proteins (SREBPs [Theopold et al., 1996; Shimano, 2001; Seegmiller et al., 2002], also known as HLH106 in flies, Figure 2A,B), using a reporter subject to the same physiologically regulated proteolytic processing as wild-type SREBP (Kunte et al., 2006). SREBP activation after mating was accompanied by upregulation of midgut transcripts involved in fatty acid synthesis and activation (SREBP, the long-chain fatty acid CoA ligases bubblegum (bgm) and AcylCoA synthetase long-chain (Acsl) and, depending on genetic background, Fatty acid synthase (FAS) and Acetyl-CoA carboxylase (ACC)) (Figure 2E,F), many of which are known SREBP targets in flies and/or mammals (Seegmiller et al., 2002; Horton et al., 2003). Immunohistochemical analyses using reporters pointed to the ECs located in the posterior midgut region (R5, Buchon et al., 2013; Marianes and Spradling, 2013) as preferential sites of transcriptional and SREBP activity changes (Figure 2A-D). Thus, in female flies actively engaged in reproduction, changes in both intestinal progenitors and their progeny parallel those observed in mammals leading to hyperplasia 


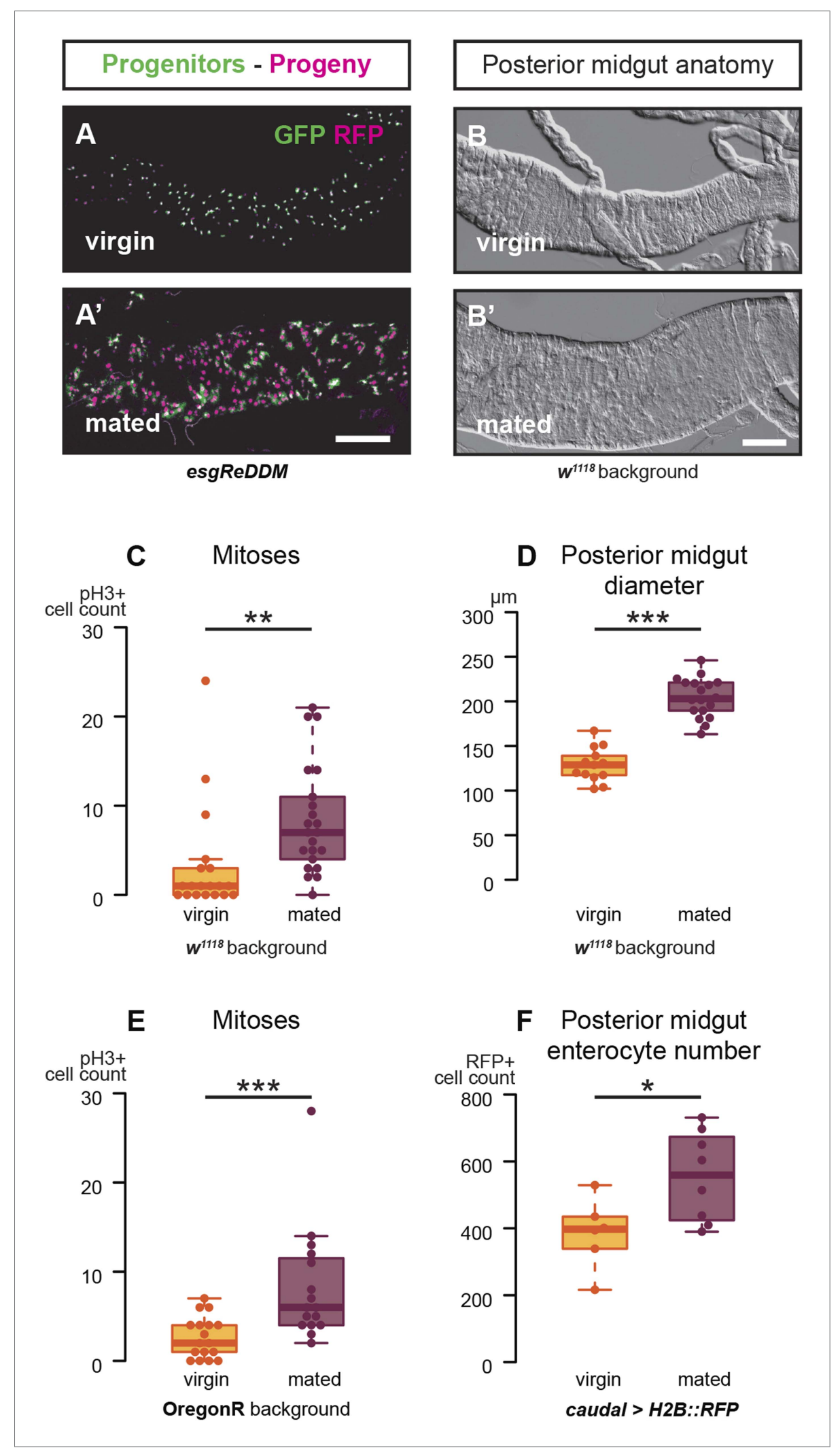

Figure 1. Mating increases ISC proliferation and gut size. (A, $\mathbf{A}^{\prime}$ ) Using the esgReDDM tracer (Antonello et al., 2015), intestinal progenitors (intestinal stem cells (ISCs) and enteroblasts) are labelled with GFP and RFP, whereas the postmitotic progeny (enterocytes (ECs) and enteroendocrine cells) that these progenitors give rise to in Figure 1. continued on next page 
Figure 1. Continued

a defined time window is labelled with RFP only (see Supplemental Information for additional details). At 3 days after mating, the posterior midgut of mated flies contains more newly generated postmitotic progeny (A) compared to age-matched virgins $\left(\mathbf{A}^{\prime}\right)$. It has also become visibly larger $\left(\mathbf{B}, \mathbf{B}^{\prime}\right)$. At this time point, these guts also have a higher number of nuclei marked by the mitotic marker $\mathrm{pH} 3$ in both $\mathrm{w}^{1118}$ and OregonR backgrounds $(\mathbf{C}, \mathrm{p}=0.008$, and $\mathbf{E}$, $p<0.001$, negative binomial GLM), although the proliferation increase is transient (data not shown). The size increase is quantified in the posterior midgut by measuring midgut diameter $(\boldsymbol{D}, p<0.001$, t-test) and counting the number of cells labelled by the EC marker caudal-Gal4 ( $F, p=0.02$, t-test). See Table 1 for full genotypes.

DOI: 10.7554/eLife.06930.003

The following figure supplement is available for figure 1:

Figure supplement 1. Mating re-sizes the Drosophila gut. DOI: 10.7554/eLife.06930.004

(Hammond, 1997; Speakman, 2008), increased organ size (Hammond, 1997; Speakman, 2008) and upregulation of lipid gene expression (Athippozhy et al., 2011).

\section{Intestinal remodelling is mediated by increased levels of circulating juvenile hormone}

Female flies change their physiology and behaviour (e.g., by increasing egg production and food intake) in response to male-derived peptides acquired during mating (Carvalho et al., 2006; Barnes et al., 2008). The synthesis of juvenile hormone (JH) in the corpus allatum, an endocrine gland, can be stimulated ex vivo by the male-derived Sex Peptide, suggesting regulation by mating (Moshitzky et al., 1996). Using rapid direct analysis in real time (DART) mass spectrometry, we profiled haemolymph of both virgin and mated female flies and established that the levels of in vivo circulating $\mathrm{JH}$ are indeed increased after mating (Figure 3A). The levels of two other juvenoid compounds, JH3-bisepoxide (JH3B) and methylfarnesoate (MF), were too low to be detected. We cannot, however, rule out that they also are regulated by mating and contribute to signalling through the $\mathrm{JH}$ pathway (Yin et alı, 1995; Tiu et al., 2012; Wen et alı, 2015). JH has been shown to sustain ovarian maturation through pleiotropic actions on adipose and reproductive tissues (Flatt et al., 2005), but its intestinal roles remain to be established. Consistent with a possible intestinal role, we detected transcript upregulation of the $\mathrm{JH}$ target Kruppel homolog 1 (Kr-h1) (Jindra et al., 2013) in guts of mated females (Figure 3-figure supplement 1). To explore the roles of JH signalling on intestinal remodelling, we first fed methoprene, a JH analogue (JHa) (Cerf and Georghiou, 1972), to virgin female flies. This led to effects on intestinal progenitors, gut diameter, and lipid metabolism comparable to those triggered by mating (Figure 3B-E,H, Figure 3-figure supplement 1). We next blocked endogenous $\mathrm{JH}$ production by mis-expressing the protein phosphatase inhibitor NiPp1 using the corpus allatum-specific driver Aug21-Gal4 (Siegmund and Korge, 2001): a genetic manipulation known to result in adult-specific ablation of the corpus allatum and a dramatic reduction of $\mathrm{JH}$ titres in the haemolymph (Yamamoto et al., 2013). Depletion of systemic JH prevented mating-triggered remodelling: a phenotype that could be restored in these gland-ablated flies by JHa feeding (Figure 3F,I).

\section{JH signals directly to adult intestinal progenitors and enterocytes via Met and Gce receptors}

To establish the cellular targets of JH and its mode of action, we interfered with $\mathrm{JH}$ signalling in a cellautonomous manner in the intestine. We used the esgReDDM system, based on the widely used esgGal4 driver (Micchelli and Perrimon, 2006), to target both classes of intestinal progenitor cells (ISCs and EBs). We first confirmed that expression of RNAi transgenes against either of the two previously identified JH receptors Methoprene-tolerant (Met) or germ cell-expressed bHLH-PAS (gce) (Jindra et al., 2013; Jindra et al., 2015) resulted in a significant reduction in their transcript levels (Figure 3-figure supplement 1). We then confined expression of these RNAi transgenes against Met, gce, or their target $\mathrm{Kr}$-h1 to adult intestinal progenitors using esgReDDM. Downregulation of any of these three genes fully prevented both the proliferative response to mating and midgut resizing, whereas overexpression of $\mathrm{Kr}$-h1 led to mating-like responses in virgin females (Figure 3G,J, 


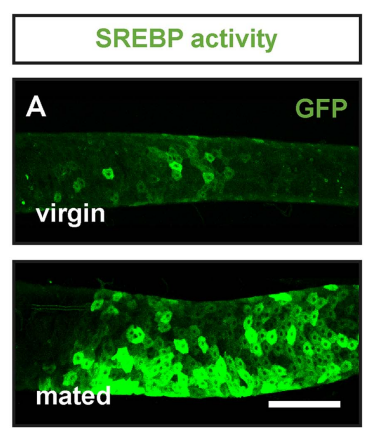

SREBP > CD8::GFP

\section{B SREBP activity}

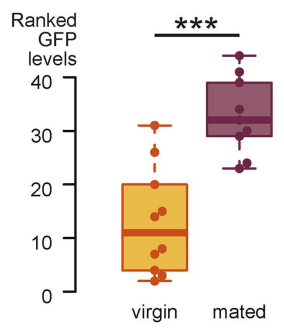

SREBP > CD8::GFP

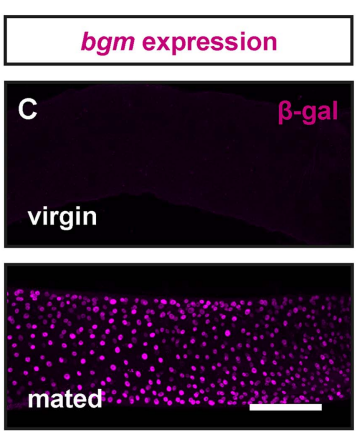

bgm-lacZ
D bgm expression

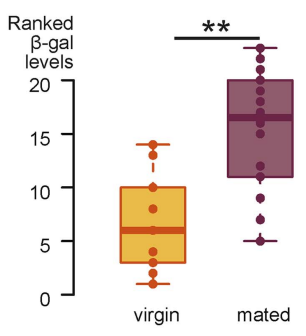

bgm-lacZ
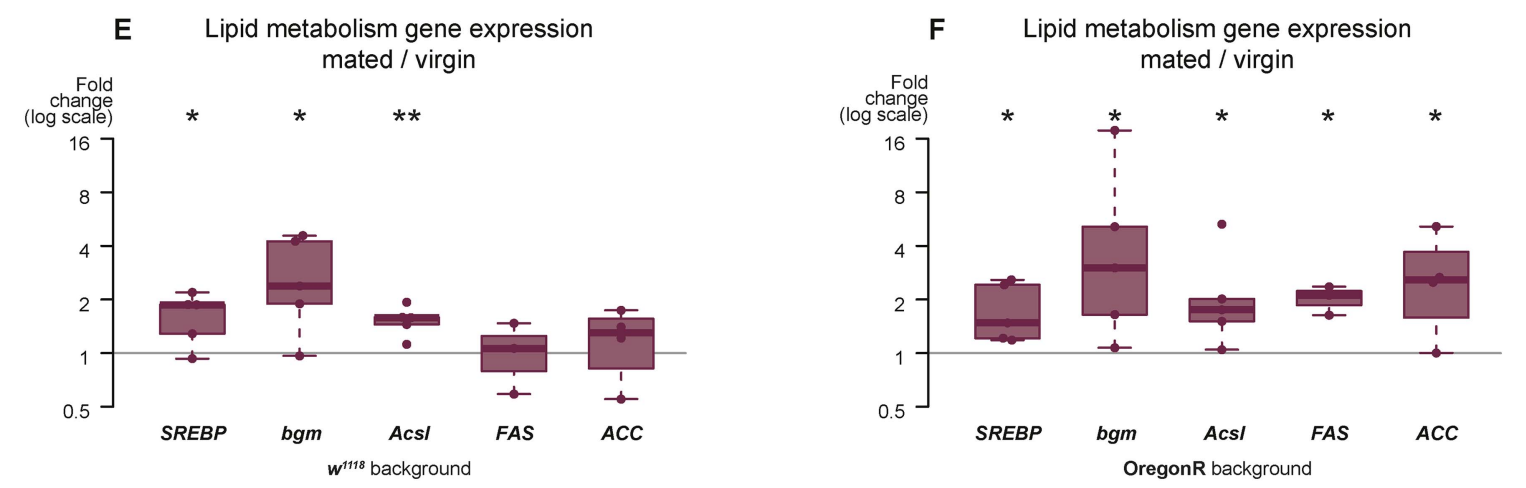

Figure 2. Mating changes the activity and/or expression of lipid metabolism genes in the intestine. At 3 days after mating, increased expression of a reporter that replicates the transcriptional regulation and post-translational modification of sterol regulatory element-binding protein (SREBP) is apparent in the posterior midgut ( $\mathbf{A}, \mathbf{A}^{\prime}$, quantified in $\mathbf{B}, p<0.001$, Mann-Whitney test). A bgm transcriptional reporter is also increased specifically in the ECs of the posterior midgut following mating $\left(\mathbf{C}, \mathbf{C}^{\prime}\right.$, quantified in $\mathbf{D}, \mathrm{p}=0.002$, Mann-Whitney test). Transcript abundance of SREBP, bgm, and the SREBP targets Acyl-CoA synthetase long-chain (Acsl), Fatty acid synthase (FAS), and Acetyl-CoA carboxylase (ACC) is increased by mating in either one or both of the $w^{1118}$ and OregonR backgrounds $\left(E w^{1118}: p=0.02\right.$ SREBP, $p=0.02$ bgm, $p=0.005$ Acsl, $p=0.5$ FAS, $p=0.3$ ACC; F OregonR: $p=0.02$ SREBP, $p=0.03$ bgm, $p=0.03$ Acsl, $p=0.01$ FAS, $p=0.04$ ACC, paired one-tailed t-test). See Table 1 for full genotypes.

Figure 3-figure supplement 1), indicating direct actions of JH on intestinal progenitors. Intestinal progenitors with downregulated $\mathrm{JH}$ receptors were found in numbers comparable to those of controls in virgin females and were able to increase their proliferation in response to a $\mathrm{JH}$-unrelated stimulus: the ROS-inducing compound paraquat (Biteau et al., 2008) (Figure 3-figure supplement 1). This indicates that they remain competent to respond to the well-studied homeostatic machinery that maintains gut integrity (Jiang and Edgar, 2012), and suggests that mating- and damage-induced proliferative mechanisms may differ and can be uncoupled. In ECs, targeted by the specific driver Mex-Gal4 (Phillips and Thomas, 2006), only downregulation of gce strongly reduced the matingdependent upregulation of a bgm reporter (Figure 3K, Figure 3-figure supplement 1). Together, these findings show that intestinal remodelling results from a rise in systemic $\mathrm{JH}$ triggered by mating. $\mathrm{JH}$ signals directly to intestinal progenitors to yield a larger organ in a Met and gce-dependent manner. Acting predominantly through gce, JH also adjusts gene expression in ECs to support increased lipid metabolism.

\section{Mating-triggered intestinal remodelling enhances reproductive output}

Intestinal remodelling during reproduction could result from increased nutrient intake (O'Brien et al., 2011) or utilisation by the developing offspring. Alternatively, it may occur in preparation for, but be uncoupled from, such nutritional demands. Consistent with the latter idea, the mating-triggered changes in proliferation, midgut size, and SREBP activity are all still apparent in sterile female ovo ${ }^{D 1}$ 
A Juvenile hormone III levels

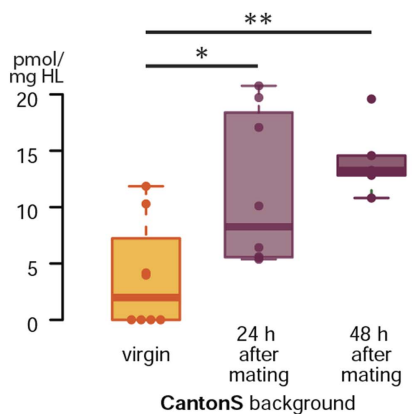

E

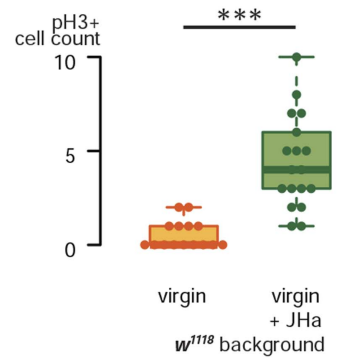

H Posterior midgut diameter

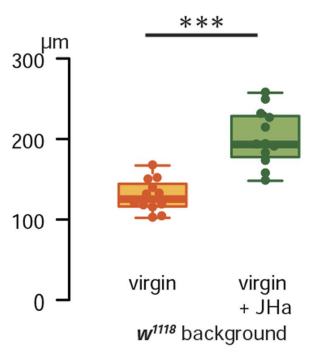

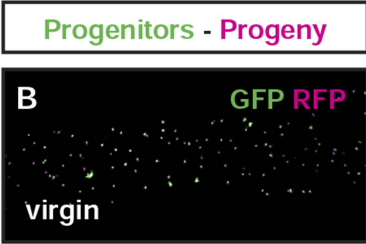

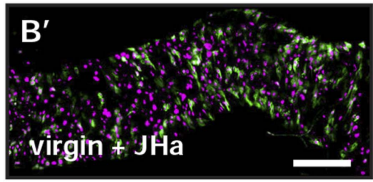

esgReDDM
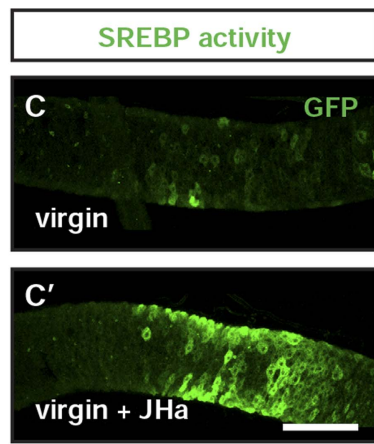

SREBP > CD8::GFP

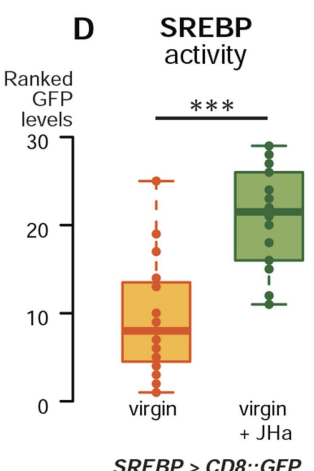

SREBP > CD8::GFP
$\mathbf{F}$

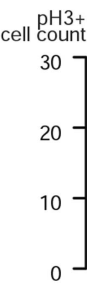

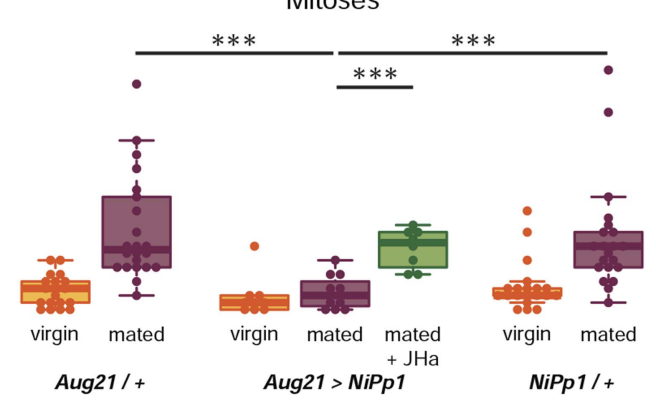

I

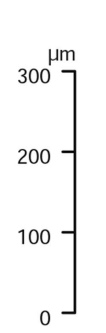

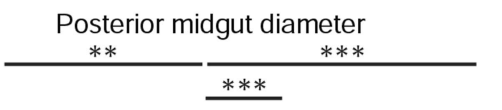
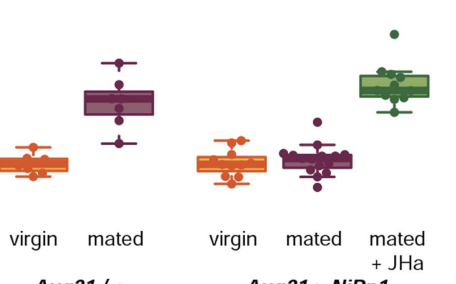

Aug21 > NiPp1

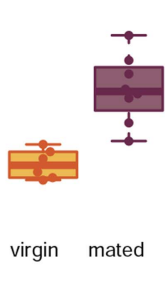

NiPp1/+
G

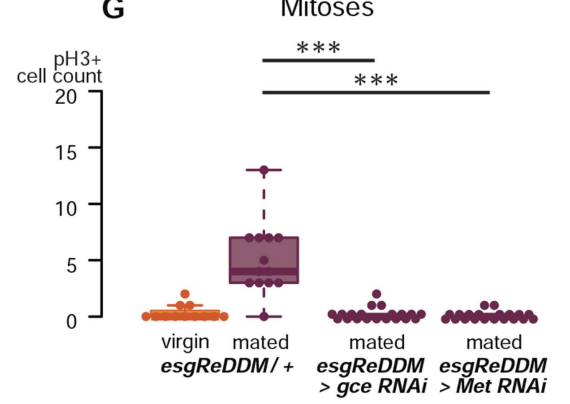

J Posterior midgut diameter

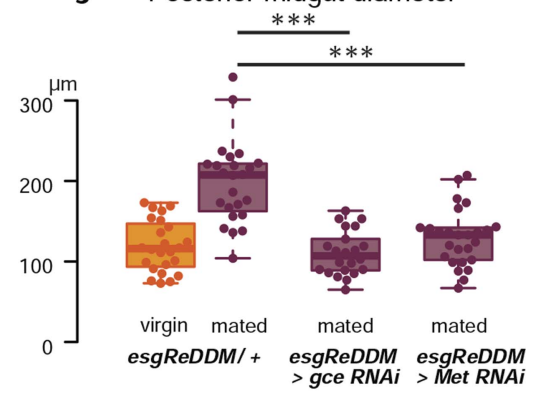

bgm expression - mated

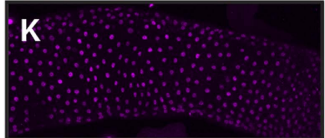

Met RNAi / +

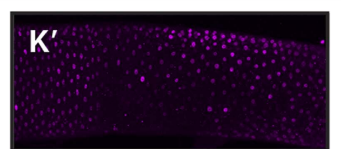

Mex > Met RNAi

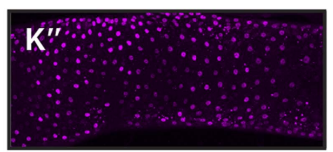

gce RNAi / +

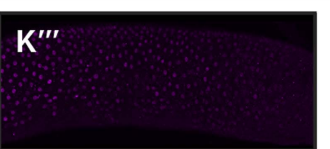

Mex > gce RNAi

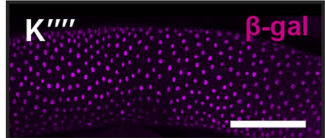

Mex / KK control

Figure 3. Systemic JH secreted after mating acts directly in the intestinal epithelium to drive reproductive remodelling. Circulating juvenile hormone (JH) is elevated after mating in the haemolymph of female flies (A, p = 0.02 at $24 \mathrm{hr}, \mathrm{p}=0.002$ at $48 \mathrm{hr}$, t-test with Holm's correction). Increased tissue renewal $\left(\mathbf{B}, \mathbf{B}^{\prime}\right)$ and SREBP activation $\left(\mathbf{C}, \mathbf{C}^{\prime}\right.$, quantified in $\mathbf{D}, \mathbf{p}<0.001$, Mann-Whitney test) are apparent following a 3-day dietary supplementation with $\mathrm{JH}$ analogue ( $\mathrm{JHa}$ ). JHa treatment is sufficient to increase mitoses $(\mathbf{E}, \mathrm{p}<0.001$, negative binomial $\mathrm{GLM})$ and size $(\mathbf{H}, \mathrm{p}<0.001$, t-test) of the posterior midgut. Conversely, when the endogenous JH source is genetically ablated by means of Aug21 > NiPp1 (Yamamoto et al., 2013), the proliferation and size increase that follow mating are abolished, although they can be reinstated by feeding JHa (proliferation $\mathbf{F}, \mathrm{p}<0.001$ between Aug21/+ and Aug21 > NiPp1 mated, p < 0.001 between Aug21 > NiPp1 and NiPp1/+ mated, p < 0.001 between Aug21 > NiPp1 and Aug21 > NiPp1 + JHa mated; all relevant comparisons between virgins are not significant, negative binomial GLM with Holm's correction; gut diameter I, $p=0.002$ between Aug21/+ and Aug21 > NiPp1 mated, $\mathrm{p}<0.001$ between Aug21 > NiPp1 and NiPp1/+ mated, $\mathrm{p}<0.001$ between Aug21 > NiPp1 and Aug21 > NiPp1 + JHa mated; all relevant comparisons between virgins are not significant, t-test with Holm's correction). Downregulation of either gce or Met in adult progenitors abrogates post-mating proliferation (G, $\mathrm{p}<0.001$ between esgReDDM/+ and esgReDDM $>$ gce RNAi mated, $\mathrm{p}<0.001$ between esgReDDM/+ and esgReDDM > Met RNAi mated, negative binomial GLM with Holm's correction) and gut size increase (J, $p<0.001$ between esgReDDM/+ and esgReDDM $>$ gce RNAi mated, $\mathrm{p}<0.001$ between esgReDDM/+ and esgReDDM > Met RNAi mated, t-test with Holm's correction). The upregulation of bgm Figure 3. continued on next page 
Figure 3. Continued

reporter upon mating is abolished by the downregulation of gce, but not Met, in ECs using the EC-specific driver Mex-Gal4 (K-K'"'). See Table 1 for full genotypes.

DOI: 10.7554/eLife.06930.006

The following figure supplement is available for figure 3 :

Figure supplement 1. Intestinal JH signalling is relayed through $\mathrm{Kr}$-h1 and underlies mating-dependent intestinal growth and gene expression phenotypes.

mutant flies in which egg production is blocked prior to vitellogenesis and which do not increase food intake after mating (Barnes et al., 2008) (Figure 4-figure supplement 1). To investigate the significance of intestinal remodelling, we used several RNAi transgenes to downregulate either the JH receptors or SREBP, which is activated by mating, specifically in adult ECs. In all cases, EC-specific downregulation led to a reduction in the number (but not viability) of eggs produced (Figure $4 E, F$ and Figure 4-figure supplement 1), indicating that $\mathrm{JH}$ signalling is required to specifically enhance the quantity (fecundity), but not the quality (viability), of reproductive output. Progenitor cell-specific downregulation may also be expected to reduce fecundity; however, we detected expression of several intestinal progenitor drivers outside the intestine (data not shown), which could affect egg production independently of the intestine. More specific tools will be necessary to resolve this important issue.

The anatomical proximity between the ovary and the posterior midgut region where changes in lipid gene expression and activity take place (Figure $4 A, D$ ) raises the intriguing possibility that enhanced nutrient delivery from the intestine to the ovary may occur locally, to maximise loading into eggs. As the trafficked nutrients would be therefore released in the form of eggs, we used sterile ovo ${ }^{D 1}$ females to quantify lipid content, reasoning that it might accumulate in gut and/or peripheral fat stores in the absence of the local ovarian sink (Parra-Peralbo and Culi, 2011). Consistent with this idea, sterile ovo ${ }^{D 1}$ female flies accumulate peripheral fat after mating (Figure $4 B, C$ ), and lipid accumulation in the posterior midgut could be induced in fertile flies by either treatment of these sterile flies with JHa or by knocking down lipid shuttling proteins acutely, thereby blocking all lipid circulation (Palm et al., 2012) (Figure 4G-J). Together, these data show that the metabolic reprogramming of ECs by JH supports fecundity, thus confirming that intestinal plasticity is required to sustain reproductive output at the time of peak fertility. The importance of intestinal lipogenesis is becoming increasingly recognised in both flies and mammals (Lodhi et al., 2011; Palm et al., 2012; Sieber and Thummel, 2012; Song et al., 2014), and here we show that it underpins reproductive output. Notably, upregulation of SREBP target genes has been reported in the small intestine of lactating rats (Athippozhy et al., 2011), suggesting that our findings may be conserved beyond insects.

\section{Discussion}

\section{Intestinal remodelling and the costs of reproduction}

The onset of reproduction involves a significant shift in metabolic demands, now routed towards the growing offspring as well as the mother. Drosophila may experience a particularly extreme example of this shift after mating: an event that enhances egg production tenfold and triggers multiple metabolic and behavioural adaptations (Kubli, 2003; Rogina et al., 2007; Avila et al., 2011; Cognigni et al., 2011). These changes are in large part brought about by signals delivered by the male during copulation, in particular the Sex Peptide (Kubli, 2003; Avila et al., 2011). Several reports connect SP to the corpus allatum and JH production (Moshitzky et al., 1996; Bontonou et al., 2015), suggesting that the systemic effects of mating via SP could be carried out through this pathway. Intriguingly, both JH knockdown in females (Yamamoto et al., 2013) and SP deficiency in males (Wigby and Chapman, 2005) extend female lifespan while reducing reproductive output and/or peak fertility. This 'cost of mating' on lifespan is not relieved by sterility (Ueyama and Fuyama, 2003), suggesting that physiological effects in non-reproductive tissues are responsible. The intestinal reprogramming that we describe here represents a novel physiological target of postmating plasticity ideally placed at the interface between nutrition and reproduction. Ageing in flies is accompanied by reduced fertility 
A Lipid-containing sites in the female abdomen
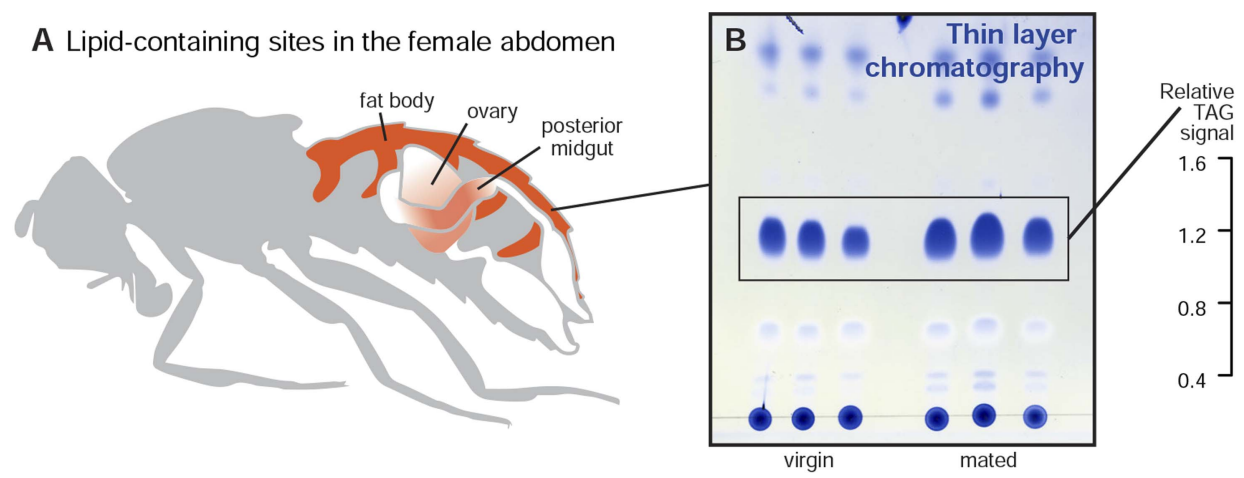

Anatomy

muscle - SREBP activity

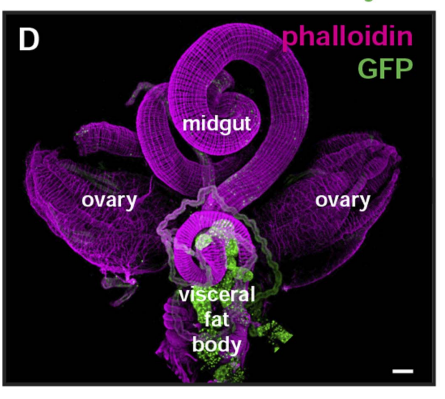

SREBP > CD8::GFP

\section{E}

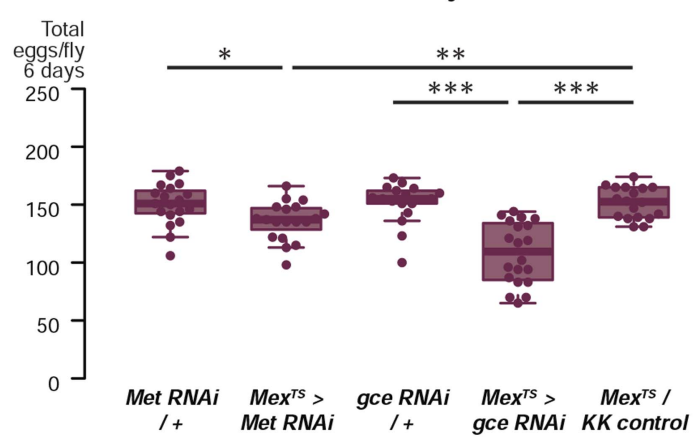

C

Triglyceride content

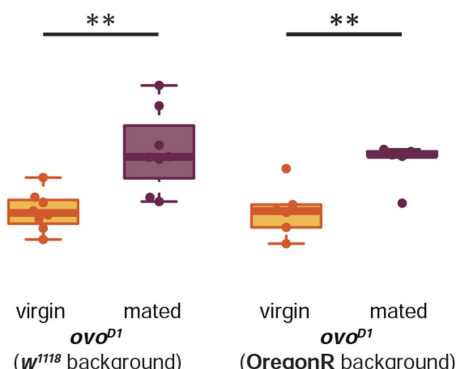

Neutral lipid distribution posterior midgut
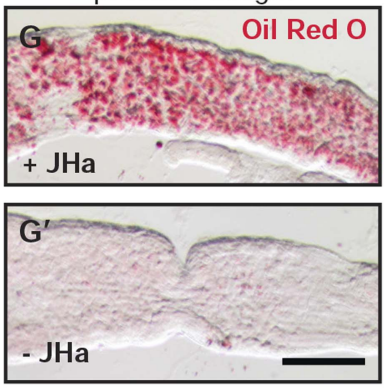

ovo $o^{D 1}$ (OregonR background)
H Neutral lipid accumulation

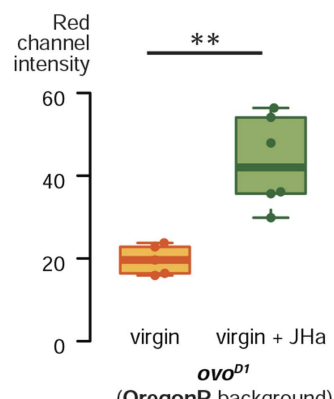

(OregonR background)
Neutral lipid distribution posterior midgut
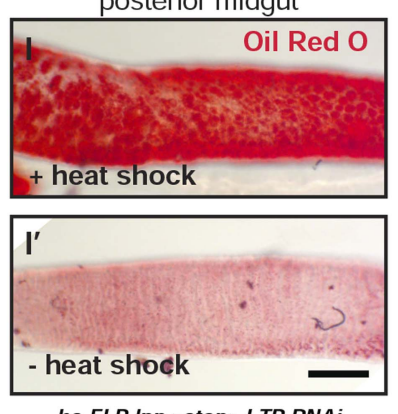

$h s-F L P$ Ipp $>$ stop $>$ LTP RNAi

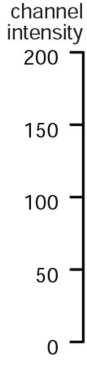

$\mathbf{F}$

Fecundity

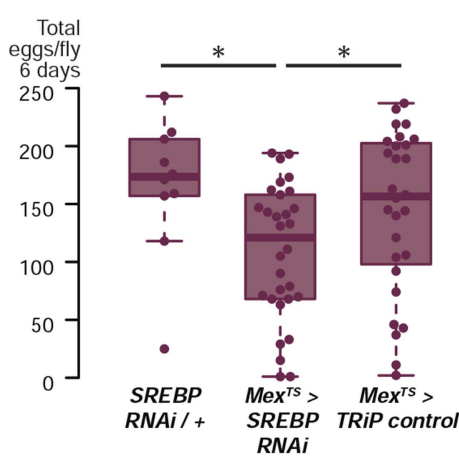

J

Neutral lipid accumulation
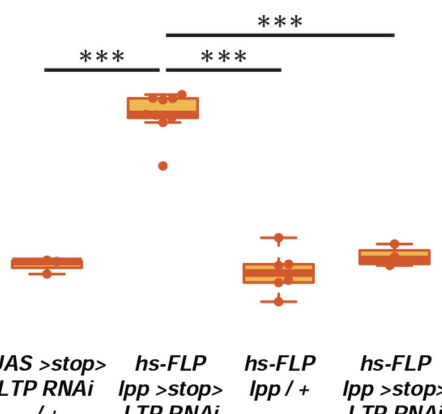

$\begin{array}{lccc}\text { UAS >stop } & h s-F L P & h s-F L P & h s-F L P \\ \text { LTP RNAi } & \text { Ipp >stop> } & \text { Ipp / } & \text { Ipp }>\text { stop }\end{array}$

I+ LTPRNAi LTPRNAi

no heat shock

Figure 4. Metabolic remodelling of ECs by JH sustains reproduction. Lipid-harbouring tissues (fat body, posterior midgut, and ovary) are found in close proximity in the fly's abdomen (represented schematically in $\mathbf{A}$, and in confocal microscopy in $\mathbf{D}$ ). The amount of stored triglycerides (TAG) in the carcass of 3-day mated sterile female flies is increased compared to virgins ( $\mathbf{B}, p=0.003$ in $w^{1118}, p=0.009$ in OregonR, t-test), as quantified by thin-layer chromatography $(\mathbf{C})$. Adult-specific downregulation of JH receptors gce and Met or SREBP in ECs reduces the total progeny produced by females in the 6 days following their first mating $\left(\mathbf{E}, \mathrm{p}=0.01\right.$ between Met RNAi/+ and Mex ${ }^{\text {ts }}>$ Met RNAi, $\mathrm{p}=0.007$ between Mex ${ }^{\text {ts }}>M e t$ RNAi and Mex ${ }^{\text {ts }} / K K$ control, $\mathrm{p}<0.001$ between gce RNAi/+ and Mex ${ }^{\text {ts }}>$ gce RNAi, $\mathrm{p}=0.007$ between Mex ${ }^{\text {ts }}>$ gce RNAi and Mex $/$ KK control; F p = 0.04 between SREBP RNAi/+ and Mex ${ }^{\text {ts }}>$ SREBP RNAi, $\mathrm{p}=0.04$ between Mex ${ }^{\text {ts }}>$ SREBP RNAi and Mex $x^{\text {ts }} /$ TRiP control, t-test with Holm's correction). In the absence of the ovarian lipid sink in sterile ovo ${ }^{D 1}$ virgin flies, treatment with $\mathrm{JHa}$ increases neutral lipid content, as revealed by Oil Red O staining, in the posterior midgut (G, G', quantified in $\mathbf{H}: p=0.002$, t-test). Acute block of lipid export by heat-shock activation of lpp > stop > LTP RNAi (Palm et al., 2012) in virgin females results in heavy accumulation of neutral lipid in this gut region, further indicating that this midgut region provides a net source of lipid in adult flies $(\mathbf{I}$, quantified in $\mathbf{J}: \mathbf{p}<$ 0.001 between LTP RNAi/+ and lpp > stop > LTP RNAi, p < 0.001 between lpp > stop > LTP RNAi and lpp > stop $>/+$, p < 0.001 between lpp $>$ stop $>$ LTP RNAi and Ipp > stop > LTP RNAi heat shock control, t-test with Holm's correction). See Table 1 for full genotypes.

DOI: 10.7554/eLife.06930.008

The following figure supplement is available for figure 4:

Figure supplement 1. Reproductive intestinal remodelling is uncoupled from germline demands and is needed to sustain reproduction. DOI: 10.7554/eLife.06930.009 
(Economos et al., 1979) and intestinal dysplasia (Biteau et al., 2008; Choi et al., 2008), and genetic manipulations that affect intestinal progenitors can affect lifespan (Biteau et al., 2010; Rera et al., 2011). Thus, it will be informative to explore the links between JH-triggered postmating responses in lifespan, age-dependent intestinal and reproductive dysfunction, and lifetime fertility.

\section{Hormonal remodelling of adult organs}

In larvae, the corpora allata integrate age and metabolic status information to optimise developmental progression (Mirth and Shingleton, 2012; Sarraf-Zadeh et al., 2013). Increasing evidence is revealing that, in adults, this insect endocrine organ acts as a nexus that detects changes in the organism's circumstances and condition to alter its metabolic and/or reproductive set points. It does so through regulated release of $\mathrm{JH}$, with pleiotropic effects on ovarian maturation, adipose tissue, learning and memory, diapause, innate immunity, and ageing (Nijhout and Riddiford, 1974; Jowett and Postlethwait, 1980; Fahrbach and Robinson, 1996; Wyatt and Davey, 1996; Flatt et al., 2005; Riddiford, 2012; Yamamoto et al., 2013). Some of these effects may be modulated by crosstalk between JH and other systemic signals such as insulin-like peptides and ecdysteroids (Jindra et al., 2013; Koyama et al., 2013; Mirth et al., 2014; Rauschenbach et al., 2014), but the cellular and molecular targets of $\mathrm{JH}$ action remain incompletely understood. Our findings have uncovered a direct and functionally significant effect on adult organ plasticity by showing that $\mathrm{JH}$ promotes proliferation and resets gut size through its actions on intestinal progenitors and activates expression of lipid metabolism genes in ECs. Based on structural and functional similarities, insect JH has been compared to mammalian thyroid hormones (Flatt et al., 2006): key energy balance regulators often associated with gastrointestinal alterations when pathologically dysregulated (Middleton, 1971). Given the well-established changes in thyroid function during human pregnancy (Glinoer, 1997), it will therefore be of interest to explore their contribution to reproductive intestinal remodelling. Downstream of its receptor(s), relay of $\mathrm{JH}$ signalling in the intestine may differ from the classical model in which Met and gce act redundantly (Abdou et al., 2011; Jindra et al., 2013). Indeed, downregulation of either gene alone is sufficient to prevent the mating-induced changes in intestinal progenitors: a finding that we confirmed by observing that viable Met $^{27}$ mutants also fail to undergo mating-induced remodelling (data not shown). The actions of Met and Gce may also be cell-specific, as suggested by a preferential requirement for gce in ECs, and may result from different Met/gce expression levels (our unpublished observations) and/or interacting partners. Candidates to consider include Taiman, homologous to the mammalian steroid receptor coactivator 1 (SRC-1)/NCoA-1/p160 (Charles et al., 2011; Li et al., 2011; Zhang et al., 2011) and, more intriguingly, circadian clock proteins: Met-binding partners recently shown to coordinate the switch from diapause to reproduction in other insects (Shin et alı, 2012; Bajgar et al., 2013).

\section{Adult organ plasticity, obesity, and cancer}

Adult organ plasticity is not a peculiarity of Drosophila reproduction; examples of changes in intestinal size and nutrient utilisation are widespread across the animal kingdom in response to both environmental and internal challenges (Carey, 1990; Hammond, 1997; Piersma and Lindstrom, 1997; Speakman, 2008; O'Brien et al., 2011). Although intestinal remodelling has not been assessed in human pregnancy, it could be one of the major drivers for the changes in gut microbiota observed during pregnancy (Koren et al., 2012) and could contribute to changes in gastrointestinal physiology, common during pregnancy (Keller et al., 2008). Resetting of anatomical or metabolic features of internal organs may thus be a broadly used strategy to achieve a positive energy balance which, when matched to the developing offspring's demands, will contribute to reproductive success. However, if deployed in the absence of such demands, organ remodelling could contribute to the weight gain and increased fat mass that has been observed upon gonadectomy of multiple species including mice, rats, cats, monkeys, and other mammals (Hansen et al., 2013 and references therein). In a more physiological context, inappropriate persistence of such metabolic remodelling beyond pregnancy and lactation could similarly contribute to post-pregnancy weight retention in humans-a phenotype that, at least in mice, is correlated with enhanced intestinal function (Casirola and Ferraris, 2003; Gore et al., 2003). Similarly, inappropriate persistence of $\mathrm{JH}$-like mechanisms that change the homeostatic set point of adult stem cells and their progeny to transform an organ may also help explain why pregnancy changes the susceptibility to certain cancers (Gwinn et al., 1990). 


\section{Materials and methods}

\section{Fly strains}

For wild-type experiments, the genetic backgrounds $w^{1118}$, OregonR, and CantonS were used as indicated in the figures and/or full genotypes list (Table 1). The following transgenic and mutant stocks were used: esg-Gal4 (Bloomington, unknown insertion), tub-Gal80 (Bloomington 7018, McGuire et al., 2003), UAS-mCD8::GFP (Bloomington 5130, Lee and Luo, 1999), UAS-H2B::RFP (presumed from Langevin et al., 2005), caudal-Gal4 (insertion used in Ryu et al., 2008), SREBP-Gal4 (Bloomington 38395, Kunte et al., 2006), bgm-lacZ (Bloomington 28120, Min and Benzer, 1999), Aug21-Gal4 (Bloomington 30137, Siegmund and Korge, 2001), UAS-NiPp1 (Bloomington 23712, Parker et al., 2002), tub-Gal4 (Bloomington 5138, Lee and Luo, 1999), Mex-Gal4 (Phillips and Thomas, 2006), UAS-Kr-h1 (DGRC 120052, referred to as UAS-Kr-h1), ovo D1 (Busson et al., 1983), hsFLP; Ipp-Gal4 and UAS > stop > LTP RNAi stocks (both from Palm et al., 2012). RNAi constructs were obtained from VDRC for gce (KK101814, GD11178 and GD47465), Met (KK100638), Kr-h1 (KK107935), and SREBP (GD37641 and GD37640), as well as the genetically matched KK control (KK60100); and from the Bloomington TRiP collection for SREBP (34073) and the genetically matched TRiP control (GFP in valium10, 35786). Because the control stocks are generated in the same background as the RNAi lines used, the Gal4 parental control (e.g., yv; Mex-Gal4/+; tub-Ga80 ${ }^{\text {ts }} /$ UAS-GFP) is genetically matched to the experimental genotype (e.g., yv; Mex-Gal4/+; tub-Gal80 ${ }^{\text {ts }} /$ UAS-SREBP RNAi TRiP). The line referred to as UAS-Kr-h1 $1_{G S}$ is GS(2)73ES2b, which was isolated in a genetic screen for enhancer/suppressors of a large-eye phenotype caused by DI overexpression in the Dominguez lab. Genomic DNA flanking the P-element insertions in the GS(2)73ES2b stock were recovered by inverse PCR and sequenced. A BLAST search with the obtained sequence produced perfect matches to the genomic region upstream of the Kr-h1 gene (26B5 Chromosome 2L: 6,082,603,...,6,096,498).

\section{Fly husbandry}

Fly stocks were reared on a standard cornmeal/agar diet (5.5\% cornmeal, $6 \%$ dextrose, $1.3 \%$ yeast, $0.55 \%$ agar supplemented with $0.18 \%$ nipagin and $2.9 \mathrm{ml} / \mathrm{l}$ propionic acid) or 'Iberian' diet $(4.4 \%$ wheat flour, $6 \%$ brown sugar, $3 \%$ yeast, $1 \%$ agar supplemented with $0.04 \%$ nipagin and $7.6 \mathrm{ml} / \mathrm{l}$ of propionic acid). All experimental flies were kept at $25^{\circ} \mathrm{C}$ expect for those containing temperaturesensitive regulation (tub-Gal80 ts), which were set up at $18^{\circ} \mathrm{C}$ (restrictive temperature) and transferred to $29^{\circ} \mathrm{C}$ (permissive temperature) at the time when activation was needed in the specific experiment. For all experiments, experimental and control flies were handled in parallel and experienced the same temperature shifts and treatments.

For the analysis of mating and JHa effects, virgin female flies were collected at eclosion, aged for 4-5 days on standard food and then transferred for 3 days (7 days for flies harbouring the esgReDDM transgenes, as these flies show a delay in mating responses at $29^{\circ} \mathrm{C}$ ) into new tubes in the presence of wild-type males (typically 4-5 females + 6 males) or food supplemented with $1.5 \mathrm{mM}$ methoprene (Sigma-Aldrich, St Louis, MO, PESTANAL 33375, racemic mixture), added to freshly prepared food when still liquid but $<50^{\circ} \mathrm{C}$. This concentration was chosen in a pilot dilution test from 0.5 to $7.5 \mathrm{mM}$ as the one that induced activation of the SREBP-Gal4 reporter to levels comparable to mating, and corresponds to approximately half of the concentration used in a previous study (Flatt and Kawecki, 2007). Controls were age-matched virgin females, also transferred to new tubes for the same time but without the addition of males and/or methoprene.

For the paraquat experiments, virgin female flies were raised at $18^{\circ} \mathrm{C}$ and aged for $4-5$ days after eclosion, at which point they were starved for $4 \mathrm{hr}$ without water. The flies were then transferred to vials containing filter paper soaked in $5 \%$ sucrose with or without $10 \mathrm{mM}$ paraquat dichloride (SigmaAldrich). After spending $24 \mathrm{hr}$ at $29^{\circ} \mathrm{C}$ in these vials, their midguts were dissected and stained for $\mathrm{pH} 3$ as described before.

\section{Antibodies}

The following antibodies were used: rabbit anti-pH3 (1:2000, Upstate, Merck-Millipore, Germany), sheep anti-GFP (1:1000, Biogenesis, for esgReDDM staining), goat anti-GFP (1:1500, Abcam, UK, for SREBP > mCD8::GFP staining), rabbit anti- $\beta$-galactosidase (1:5000, MP Biomedicals, Santa Ana, CA); secondary antibodies were either FITC/Cy3 conjugates from Jackson ImmunoResearch (1:200, West 
Table 1. Full genotypes

\begin{tabular}{|c|c|}
\hline Genotype in text/figure & Full genotype \\
\hline esgReDDM & w; esg-Gal4, UAS-mCD8::GFP/+; tub-Gal80ts, UAS-H2B::RFP/+; + \\
\hline$w^{1118}$ background & $w^{1118} ;+;+;+$ \\
\hline OregonR background & $+;+;+;+$ \\
\hline caudal > H2B::RFP & w; caudal-Gal4/+; UAS-H2B::RFP/+; + \\
\hline SREBP > CD8::GFP & W/+; SREBP-Gal4/+; UAS-CD8::GFP/+; + \\
\hline bgm-lacZ & $w /+;$ bgm-lacZ/+; +; + \\
\hline CantonS background & $+;+;+;+$ \\
\hline Aug21/+ & w; Aug21-Gal4/+; +; + \\
\hline Aug21 > NiPp1 & w; Aug21-Gal4/+; UAS-NiPp1/+; + \\
\hline NiPp1/+ & w; +; UAS-NiPp1/+; + \\
\hline esgReDDM/+ & w; esg-Gal4, UAS-mCD8::GFP/+; tub-Gal80"s, UAS-H2B::RFP/+; + \\
\hline esgReDDM > gce RNAi & $\begin{array}{l}\text { w; esg-Gal4, UAS-mCD8::GFP/UAS-gce RNAi KK101814; tub-Gal80"s, } \\
\text { UAS-H2B::RFP/+; + }\end{array}$ \\
\hline esgReDDM > Met RNAi & $\begin{array}{l}\text { w; esg-Gal4, UAS-mCD8::GFP/UAS-Met RNAi KK100638; tub-Gal80"s, } \\
\text { UAS-H2B::RFP/+; + }\end{array}$ \\
\hline esgReDDM > Kr-h1 RNAi & $\begin{array}{l}\text { w; esg-Gal4, UAS-mCD8::GFP/UAS-Kr-h1 RNAi KK107935; tub-Gal80ts, } \\
\text { UAS-H2B::RFP/+; + }\end{array}$ \\
\hline esgReDDM > Kr-h1 $1_{G S}$ & $\begin{array}{l}\text { w; esg-Gal4, UAS-mCD8::GFP/UAS-Kr-h1 }{ }_{G s} ; \text { tub-Gal80 }{ }^{t s}, \\
\text { UAS-H2B::RFP/+; + }\end{array}$ \\
\hline esgReDDM $>K_{r}$-h1 UAS & $\begin{array}{l}\text { W; esg-Gal4, UAS-mCD8::GFP/UAS-Kr-h1 UAS; tub-Gal80"s, UAS-H2B:: } \\
\text { RFP/+; + }\end{array}$ \\
\hline$t_{u} b^{\text {ts }}>$ Met RNAi & $\begin{array}{l}\text { w; tub-Gal80ts/UAS-Met RNAi KK100638; tub-Gal4, } \\
\text { UAS-mCD8::GFP/+; + }\end{array}$ \\
\hline tub $^{\text {ts }}>$ gce RNAi & $\begin{array}{l}\text { w; tub-Gal80ts/UAS-gce RNAi KK101814; tub-Gal4, } \\
\text { UAS-mCD8::GFP/+; + }\end{array}$ \\
\hline tub ${ }^{\text {ts }}>$ Kr-h1 RNAi & $\begin{array}{l}\text { w; tub-Gal80ts/UAS-Kr-h1 RNAi KK107935; tub-Gal4, } \\
\text { UAS-mCD8::GFP/+; + }\end{array}$ \\
\hline tub $b^{\text {ts }}>$ SREBP RNAi GD & $\begin{array}{l}\text { w; tub-Gal80 ts/UAS-SREBP RNAi GD37640; tub-Gal4, } \\
\text { UAS-mCD8::GFP/+; + }\end{array}$ \\
\hline tub ${ }^{\text {ts }}>$ SREBP RNAi TRiP & $\begin{array}{l}\text { w; tub-Gal80 ts +; tub-Gal4, UAS-mCD8::GFP/UAS-SREBP RNAi } \\
\text { TRiP34073; + }\end{array}$ \\
\hline tub $b^{\text {ts }} /+$ & w; tub-Gal80ts/+; tub-Gal4, UAS-mCD8::GFP/+; + \\
\hline
\end{tabular}

Figure panel(s)

Figure 1A, Figure 1-figure supplement $1 C, D$, Figure $3 B$

Figure $1 B-D$, Figure $2 E$, Figure $3 E, H$, Figure 3-figure supplement $1 \mathrm{H}$, Figure 4-figure supplement 1A,B

Figure 1E, Figure 1-figure supplement $1 A, B$, Figure 2F, Figure 3-figure supplement $1 \mathrm{H}$

Figure 1F, Figure 3-figure supplement 1A

Figure $2 A, B$, Figure $3 C, D$, Figure $4 D$,

Figure 4-figure supplement 1D

Figure 2C, D

Figure $3 \mathrm{~A}$

Figure $3 F$, I

Figure 3F, I

Figure $3 F, I$

Figure 3G,J, Figure 3-figure supplement $1 D, F, G, I, J$

Figure 3G,J, Figure 3-figure supplement $1 C-G$

Figure 3G,J

Figure 3-figure supplement $11, \mathrm{~J}$

Figure 3-figure supplement 1I

Figure 3-figure supplement 1I,J

Figure 3-figure supplement 1B

Figure 3-figure supplement 1B

Figure 3-figure supplement $1 \mathrm{~K}$

Figure 3-figure supplement $1 \mathrm{~K}$

Figure 3-figure supplement $1 \mathrm{~K}$

Figure 3-figure supplement $1 \mathrm{~B}, \mathrm{~K}$ (control)

Met RNAi/+ $\quad$ w; bgm-lacZ/UAS-Met RNAi KK100638; +; +

Mex $>$ Met RNAi w; Mex-Gal4, bgm-lacZ/UAS-Met RNAi KK100638; +; +

Figure $3 K$, Figure 3-figure supplement $1 \mathrm{~L}$

Figure 3K, Figure 3-figure supplement $1 \mathrm{~L}$

gce RNAi/+ $\quad$ w; bgm-lacZ/UAS-gce RNAi KK101814; +; +

Figure $3 K$, Figure 3-figure supplement $1 \mathrm{~L}$

Mex > gce RNAi w; Mex-Gal4, bgm-lacZ/UAS-gce RNAi KK101814; +; +

Figure 3K, Figure 3-figure supplement $1 \mathrm{~L}$

Mex/KK control w; Mex-Gal4, bgm-lacZ/attp40; +; +

Figure 3K, Figure 3-figure supplement $1 \mathrm{~L}$

ovo $^{D 1}$ ( $w^{1118}$ background) $\quad w^{1118} ;+$ ovo $1 /+;+$

Figure 4C, Figure 4-figure supplement $1 A, B$

Table 1. Continued on next page 
Table 1. Continued

Genotype in text/figure ovo $^{D 1}$ (OregonR background)

Met RNAi/+
Mex ${ }^{\text {ts }}>$ Met RNA
Full genotype

+/w118; +; ovo $1 / 1+$; +

w; UAS-Met RNAi KK100638/+; +; +

w; Mex-Gal4/UAS-Met RNAi KK100638; tub-Gal80 ts $/+$; +
Figure panel(s)

Figure $4 C, G, H$

Figure 4E, Figure 4-figure supplement $1 \mathrm{H}$

Figure 4E, Figure 4-figure supplement $1 \mathrm{H}$

Figure 4E, Figure 4-figure supplement $1 \mathrm{H}$

gce RNAi/+ $\quad$ w; UAS-gce RNAi KK101814/+; +; +

Mex ${ }^{\text {ts }}>$ gce RNAi W; Mex-Gal4/UAS-gce RNAi KK101814; tub-Gal80ts/4; +

Mex $\quad$ w; Mex-Gal4/attp40; tub-Gal80ts $/+$; +

SREBP RNAi/+

w/y, v; +; UAS-SREBP RNAi 34073; +

Mex ${ }^{\text {ts }}>$ SREBP RNAi

w/y, v; Mex-Gal4/+; tub-Gal80'S/UAS-SREBP RNAi 34073; +

Figure 4E, Figure 4-figure supplement $1 \mathrm{H}$

Figure 4E, Figure 4-figure supplement $1 \mathrm{H}$

Figure 4F

Mex ${ }^{\text {ts }} /$ TRiP control

w/y, v; Mex-Gal4/+; tub-Gal80ts/UAS-GFP; +

Figure 4F

hs-FLP Ipp > stop > LTP RNAi w, hs-FLP/w; lpp-Gal4/+; UAS > stop > LTP RNAi/+; +

UAS > stop > LTP RNAi/+ $\quad$ W; +; UAS > stop > LTP RNAi/+; +

Figure 4F

Figure $4 \mathrm{I}, \mathrm{J}$

hs-FLP Ipp/+

w, hs-FLP/w; Ipp-Gal4/+; +; +

Figure $4 \mathrm{~J}$

esgReDDM/ovo ${ }^{D 1} \quad$ ovo 1 1/w; esg-Gal4, UAS-mCD8::GFP/+; tub-Gal80's, UAS-H2B::RFP/4; +

SREBP > CD8::GFP/ovo D1 W/+; SREBP-Gal4/+; UAS-CD8::GFP/ovo ${ }^{D 1}$; +

gce RNAi GD11178/+ $\quad$ w; UAS-gce RNAi GD11178; +; +

Mex ${ }^{\text {ts }}>$ gce RNAi GD11178 w; Mex-Gal4/UAS-gce RNAi GD11178; tub-Gal80 $5 /+$; +

gce RNAi GD47465/+ $\quad$ wi +; UAS-gce RNAi GD47465/+; +

Mex ${ }^{\text {ts }}>$ gce RNAi GD47465 w; Mex-Gal4/+; tub-Gal80ts/UAS-gce RNAi GD47465; +

Mex ${ }^{\text {ts }} /+\quad$ w; Mex-Gal4/+; tub-Gal80 ${ }^{\text {ts }}+;+$

SREBP RNAi GD37641/+ $\quad$ w; UAS-SREBP RNAi GD37641/+; +; +

Mex ${ }^{\text {ts }}>$ GD37641

w; Mex-Gal4/UAS-SREBP RNAi GD37641; tub-Gal80ts/4; +

SREBP RNAi GD37640/+

w; UAS-SREBP RNAi GD37640/+; +

$M e x^{\text {ts }}>$ RNAi GD37640

W; Mex-Gal4/+; tub-Gal80ts/UAS-SREBP RNAi GD37640; +
Figure 4J

Figure 4-figure supplement $1 C$

Figure 4-figure supplement $1 D, E$

Figure 4-figure supplement $1 F$

Figure 4-figure supplement $1 F$

Figure 4-figure supplement $1 F$

Figure 4-figure supplement $1 F$

Figure 4-figure supplement $1 F$

Figure 4-figure supplement 1G,I

Figure 4-figure supplement 1G,I

Figure 4-figure supplement 1G,I

Figure 4-figure supplement 1G,I

DOI: 10.7554/eLife.06930.010

Grove, PA, for SREBP > mCD8::GFP and bgm-lacZ) or Alexa488/647 conjugates from Invitrogen Life Technologies (1:1000, Carlsbad, CA, for esgReDDM and caudal > H2B::RFP). Preparations for proliferation analysis were counterstained with DAPI (Sigma-Aldrich) and mounted in Fluoromount-G (Southern Biotech, Birmingham, AL). Preparations for reporter analysis were mounted in Vectashield with DAPI (Vector Labs, Burlingame, CA).

\section{Proliferation and size quantifications}

Quantification of mitoses in wild-type and ovo ${ }^{D 1}$ female flies was carried out by counting individual nuclei marked by the mitotic marker pH3 using a Nikon Eclipse 90i Fluorescence microscope through a 40x objective. For the acquisition of gut images in these samples, a single $1392 \times 1040$ field was acquired posterior to the midgut-hindgut boundary using QCapture software (Qlmaging). Progeny dynamics were analysed using the esgReDDM system (Antonello et al., 2015), which has the genetic makeup esgGal4, UAS-mCD8::GFP; tub-Gal80 ts UAS-H2B::RFP. At the permissive temperature of $29^{\circ} \mathrm{C}$, the GFP reporter is expressed in esg-Gal4 positive cells (ISCs and EBs), but due to the perdurance of the RFPtagged histone H2B::RFP the esg-Gal4-negative progeny (including ECs and EECs) generated from these progenitors since the shift to permissive temperature is additionally labelled in red. To restrict progeny analysis to mating-induced changes, esgReDDM flies were maintained at $18^{\circ} \mathrm{C}$, such that Gal4 expression is suppressed by tub-Gal $80^{\text {ts }}$, and moved to $29^{\circ} \mathrm{C}$ only at the time of mating. After 3 days of mating at $29^{\circ} \mathrm{C}$, guts were dissected and stained for GFP and pH3 (the endogenous RFP signal was 
detected directly). EC number in the posterior midgut was assessed by imaging the entire gut of caudal $>$ H2B::RFP flies and counting the number of RFP-marked cells. Confocal images were obtained with a Leica TCS SP5 inverted confocal microscope using a 20x air objective for esgReDDM and a 10x air objective for caudal > H2B::RFP. Stacks were typically collected every $1 \mu \mathrm{m}$, and the images $(1024 \times$ 1024) were reconstructed using maximum projection. Bright-field images or confocal maximum projections were loaded into ImageJ (Schneider et al., 2012) and the line tool used to quantify the width of the gut across the centre of the image. ImageJ was also used to outline the guts of esgReDDM flies using the polygon tool before analysing the resulting region of interest (ROI) with a custom MATLAB (The MathWorks, Inc.) script optimised for the ReDDM method. Extended details about this analysis are available from (Antonello et al., 2015). Briefly, maximum projections were adjusted for levels and offsets and filtered to remove noise (using always the same parameters for scans within one experiment), then the area of the gut was identified by background staining and the cell nuclei by DAPI signal. The size of nuclei can be used to discriminate between diploid cells (ISCs, EBs, and EECs) and polyploid ECs. The red-labelled nuclei (persistent H2B::RFP) and green-labelled cells (mCD8::GFP) were identified by segmentation and compared to the pattern of nuclei defined by DAPI to generate a report of total ECs (large DAPI cells), total progenitors + progeny (RFP signal), total ISCs and EBs (GFP signal), and total area. The same script was also used to count the number of caudal $>H 2 B:: R F P$ cells.

\section{Analysis of reporter gene expression}

For SREBP > mCD8::GFP and bgm-lacZ experiments, confocal images were obtained with a Leica SP5 upright confocal microscope using a $20 \times$ glycerol immersion objective. A single $20 \times$ field (1024 pixels wide) immediately posterior to the midgut-hindgut boundary was acquired with a $Z$ resolution of 1.5 $\mu \mathrm{m}$. ImageJ was used to generate a maximum projection for each sample and all images pertaining to one experiment were loaded as separate layers into a single Adobe Photoshop CS6 file. The layers were then ranked blindly on the basis of their relative intensity in the relevant channel.

\section{qPCR}

To quantify mating-induced changes in gene expression, posterior midguts from at least 10 adult female flies were dissected, discarding Malphigian tubules and the hindgut. To determine the knockdown efficiency of the RNAi transgenes, tub-Gal80, tub-Gal4, UAS-GFP was used to downregulate them ubiquitously. 8-10 third instar larvae were collected from crosses kept at $21^{\circ} \mathrm{C}$ and were shifted to $29^{\circ} \mathrm{C}$ for $3 \mathrm{hr}$ to allow RNAi transgene expression. Samples (posterior midguts or whole larvae) were directly stored on dry ice and at $-80^{\circ} \mathrm{C}$ in RNAlater TissueProtect Tubes (Qiagen, the Netherlands) until total RNA was extracted using RNeasy Mini Kit (Qiagen), from which cDNAs were prepared with SuperScript First-Strand Synthesis System (Invitrogen Life Technologies) using oligo-dT primers. Quantitative PCR was performed using the SYBR Green PCR Master Mix (Applied Biosystems Life Technologies) in a 7500 Real-Time PCR System (Applied Biosystems) using the housekeeping gene rp49 as a control. All qPCRs were performed in triplicate and the relative expression was calculated using comparative $\mathrm{Ct}$ method.

Primers used:

\begin{tabular}{lll}
\hline & Forward $\mathbf{5}^{\prime} \mathbf{- \mathbf { 3 } ^ { \prime }}$ & Reverse $\mathbf{3}^{\prime} \mathbf{- \mathbf { 5 } ^ { \prime }}$ \\
\hline SREBP & GCAAAGTGCGTTGACATTAACC & AGTGTCGTGTCCATTGCGAA \\
\hline bgm & GCAATCGATTTGCGTGACCA & GGCCCAGGACGATTGTAGAG \\
\hline Acsl & CGGAGATCCGACAAAGCAGT & TGAGCACAGCTCCTCAAAGG \\
\hline ACC & GACATTCGATCGACGCCTCT & GCTTTGGCTTCTGCACTGAC \\
\hline Kr-h1 & AATTCTCCAAGGCTCGTCCC & CATGCCGCAATTGTTTTCGC \\
\hline gce & ACAATTTTATGATTCAGCCACAACC & GTTAGTGGAGGCGGAACCTG \\
\hline Met & AGCTGCGTATCCTGGACACT & TCGAGAGCTGAAACATCTCCAT \\
\hline rp49 & CCGCCGTCCTTAGATTCGC & GTTCCCTTGAGGCCGGTTT \\
\hline
\end{tabular}




\section{Detection of circulating JH hormone by DART-MS}

Haemolymph was extracted from virgin or mated females using pulled glass microcapillary needles (10 $\mu \mathrm{l}$ vol, \#2-000-010; Drummond Scientific, PA, USA). The needle tip was placed into the gap between the anepisternum and anepimeron of anesthetised flies, and haemolymph was collected using a slight vacuum $(0.2-1.0 \mathrm{mPa})$ for $\sim 30 \mathrm{~s}$. Haemolymph from 45 to 50 flies was collected in the same needle. The contents were ejected into a $0.1 \mathrm{ml}$ glass vial insert (Thermo Fisher Scientific, MA, USA) by applying pressurised air ( 5-6 kPa) with a Femtojet microinjector (Eppendorf, NY, USA), and weighed prior to extraction. $20 \mu \mathrm{l}$ of $\mathrm{MeOH}$ was added to the haemolymph followed by extraction with $20 \mu \mathrm{l}$ of hexane, repeated four times. Pooled hexane extract was evaporated under a gentle stream of $\mathrm{N}_{2}$ and reconstituted in $10 \mu$ of hexane. All extracts were prepared and measured immediately after collection.

Mass spectra were acquired with an atmospheric pressure ionisation time-of-flight mass spectrometer (AccuTOF-DART, JEOL USA, Inc.) equipped with a DART interface and operated with a resolving power of 6000 (FWHM definition). The RF ion guide voltage was set at $600 \mathrm{~V}$. The atmospheric pressure ionisation interface potentials were as follows: orifice $1=15 \mathrm{~V}$, orifice $2=5 \mathrm{~V}$, ring lens $=5 \mathrm{~V}$. Mass spectra were stored at a rate of one spectrum per second with an acquired $\mathrm{m} / \mathrm{z}$ range of 60-1000. The DART interface was operated in positive-ion mode using helium gas with the gas heater set to $200^{\circ} \mathrm{C}$. The glow discharge needle potential was set to $3.5 \mathrm{kV}$. Electrode 1 was set to $+150 \mathrm{~V}$, and electrode 2 was set to $+250 \mathrm{~V}$. Helium gas flow was set to $2.0 \mathrm{l} / \mathrm{min}$. Calibration for exact mass measurements was accomplished by acquiring a mass spectrum of polyethylene glycol (average molecular weight 600) as a reference standard in every data file. Analysis was done with JEOL MassCenter software (version 1.3.0.1). Accurate mass measures and isotope pattern matching by MassMountaineer (FarHawk Marketing Services, Ontario, CA) were used to support elemental composition assignments.

$2 \mu \mathrm{l}$ of the haemolymph hexane extract was placed on the tip of a borosilicate glass capillary. The capillary was introduced to the DART ion source with a micromanipulator, thus allowing for reproducible placement of the sample. Each extract was measured 4-5 times. The averaged signal intensity was normalised to the total weight of the haemolymph and converted to absolute quantities after establishing a calibration curve with a JHIII standard (Santa Cruz Biotechnology, CA, USA, CAS 24198-95-6). Analysis of JHIII by DART produces two signature ions at $\mathrm{m} / \mathrm{z} 267.20$ (intact molecule) and at $\mathrm{m} / \mathrm{z} 249.18$ (loss of water), consistent with a previous study (Navare et al., 2010). The abundance of the $\left[\mathrm{M}-\mathrm{H}_{2} \mathrm{O}+\mathrm{H}\right]^{+}$signal peak was used for all measurements because the parent ion at $\mathrm{m} / \mathrm{z} 267.20$ could not be consistently resolved due to interference from other signals. To detect other juvenoid compounds, the following mass signatures were used: methylfarnesoate $\left([\mathrm{M}+\mathrm{H}]^{+} 251.20\right)$ and JHIII Bisepoxide $\left([\mathrm{M}+\mathrm{H}]^{+}\right.$283.19). DART MS was previously shown to be an effective method for quantitative and high-sensitivity measurements of JHIII (Navare et al., 2010).

\section{Fecundity and egg viability experiments}

Flies for fecundity and egg viability experiments were raised at $18^{\circ} \mathrm{C}$ to prevent the expression of the RNAi transgenes during development, then shifted to $29^{\circ} \mathrm{C}$ in late pupariation (after $\sim 20$ days). Virgin females were collected upon eclosion and after 4 days mated overnight to OregonR males (10 males, 10 females per vial). Males were then removed, individual female flies were transferred to a new single vial of yeast-supplemented standard food (cornmeal/agar diet with $5 \%$ yeast content) every $48 \mathrm{hr}$, and eggs were counted from the vacated vial to quantify fecundity. To assess egg viability, a fraction of the egg-containing vials were then maintained at $29^{\circ} \mathrm{C}$, and the number of eclosed progeny was counted and compared with egg counts. Each genotype cross was performed three times, and egg production from each fly was assessed over three $48 \mathrm{hr}$ repeats, covering a total of 6 days of egg laying.

\section{Thin-layer chromatography (TLC)}

Ovaries and guts were removed from flies immobilised on ice and the remaining carcasses (three flies per sample) were immediately homogenised in a mixture of methanol $(60 \mu \mathrm{l})$, chloroform $(150 \mu \mathrm{l})$, and water $(75 \mu \mathrm{l})$, following previously described procedures (Al-Anzi et al., 2009; Hildebrandt et al., 2011). After an extraction period $\left(1 \mathrm{hr}\right.$ at $\left.37^{\circ} \mathrm{C}\right)$, aqueous and organic phases were separated by the addition of a 1:1 mixture of $1 \mathrm{M}$ potassium chloride and chloroform (75 $\mu$ l each). Samples were briefly 
centrifuged and $120 \mu \mathrm{l}$ of the organic phase was transferred to fresh Eppendorf tubes and left to air-dry for $3 \mathrm{hr}$. The resulting dessicated lipids were resuspended in $16 \mu \mathrm{l}$ of a 1:1 chloroform:methanol mixture. $3 \mu \mathrm{l}$ of each sample was applied to TLC plates (Merck Millipore116487) and lipid species were separated by standing the plates in $\sim 1 \mathrm{~cm}$ of a mobile phase consisting of $69.5 \%$ hexane, $29.5 \%$ diethyl ether, and $1 \%$ acetic acid. Once the mobile phase had traversed the plates, they were briefly dried and then dipped in a cerium-ammonium-molybdate stain (ammonium heptamolybdate tetrahydrate $2.5 \mathrm{~g}$, cerium (IV) sulphate hydrate complex with sulphuric acid $1 \mathrm{~g}$, water $90 \mathrm{ml}$, sulphuric acid $10 \mathrm{ml}$ ). The TLC plates were developed at $80^{\circ} \mathrm{C}$ for $25 \mathrm{~min}$ and then imaged on a digital scanner. The TAG content was quantified by analysing the resulting TIFF images using the densitometry tool in ImageJ software. All reagents were purchased from Sigma-Aldrich.

\section{Lipid export block}

The shuttling of lipids between organs was abolished by downregulating the apolipoprotein lipid transfer protein (LTP) through heat-shock-activated acute RNAi expression based on the pFRiPE system (Marois and Eaton, 2007). In the larva, this manipulation prevents the loading of gutoriginated medium-chain diacylglycerides, which are a dominant component of circulating lipids, onto the haemolymph carrier Lipophorin (Lpp) and leads to the accumulation of stored lipid in the larval gut in triglyceride form (Palm et al., 2012). The downregulation of LTP from the fat body driver Ipp-Gal4 was triggered in virgin females by $1 \mathrm{hr}$ heat-shock at $37^{\circ} \mathrm{C}$; after $6 \mathrm{hr}$, the guts were dissected for neutral lipid detection using Oil Red $O$ staining.

\section{Oil Red O stainings}

Fly guts were dissected from flies immobilised on ice and were then fixed in a solution of $4 \%$ formaldehyde for $45 \mathrm{~min}$. Guts were washed in consecutive applications of phosphate buffered saline (PBS), double-distilled water, and a $60 \%$ isopropanol solution. Oil Red O (Sigma-Aldrich) stock was prepared as a $0.1 \%$ solution in isopropanol, then a freshly prepared working solution (a $6: 4$ dilution in water) was added for $20 \mathrm{~min}$ to the guts, then washed in 60\% isopropanol and water. The preparations were mounted in glycerol for analysis, and the posterior midgut was imaged using either a Zeiss Axioplan stereo microscope equipped with Nomarski optics or an Olympus BX53 phase contrast microscope equipped with a $4 \times / 0.13$ UPlanFLN lens through CellSens software (Olympus, Japan). The resulting TIFF files were analysed quantitatively using a custom Image J script: the gut was manually outlined as a ROI using the polygon tool, then the RGB channels were split and the red channel subtracted from the green to eliminate background (grey) signal. The mean intensity of the resulting signal within the ROI was calculated with the built-in Analyse Particles function.

\section{Statistics and figure preparation}

All statistical analyses were carried out in the $R$ environment ( $R$ Development Core Team, 2014). Comparisons between normally distributed groups were carried out using Student's $t$-test ( $R$ function t.test), unpaired, two-tailed and incorporating Welch's correction to account for unequal variances, followed by Bonferroni-Holm correction when multiple comparisons were applied. QPCR data were analysed comparing the housekeeping-subtracted $\mathrm{Cts}$ of experimentally matched virgin and mated samples, thus using paired t-test, one-tailed when confirming previous reporter experiments (Figure 2E,F), and two-tailed when no prediction could be made (panel $\mathrm{H}$ in Figure 3-figure supplement 1). Count data with a distinctly non-normal distribution (specifically, $\mathrm{pH} 3$ counts) were fitted with a negative binomial model ( $R$ function glm.nb from MASS package, Venables and Ripley, 2002) followed by likelihood ratio tests ( $R$ function anova.negbin from MASS package). Rank-based experiments were analysed with the Mann-Whitney-Wilcoxon rank sum test (R function wilcox.test). All graphs were generated in $\mathrm{R}$ using a custom script based on the base boxplot function superimposed with individual data points plotted with the beeswarm function (package beeswarm). Confocal and bright-field images shown in conjunction were always acquired with the same settings as part of a single experiment. For visualisation purposes, level and channel adjustments were applied using Adobe Photoshop CS6 to the confocal images shown in figure panels (the same correction in all comparable images), but all quantitative analyses were carried out on unadjusted raw images or maximum projections. In all figures, ${ }^{*}$ indicates $0.05>p \geq 0.01,{ }^{* *}$ indicates $0.01>p \geq 0.001$, and *** indicates $\mathrm{p}<0.0001$. 


\section{Acknowledgements}

We thank Antoine Ducuing for his assistance with the initial SREBP experiments and Suzanne Eaton and Wilhelm Palm for sharing lipid transport data and reagents prior to publication. We are grateful to Frederic Bernard, Edward Dubrowsky, Suzanne Eaton, Marek Jindra, Christen Mirth, Isabel Palacios, Julien Royet, Alex Shingleton, and Graham Thomas for reagents. We also thank Thomas Carroll for statistical advice, and Dafni Hadjieconomou, Bruno Hudry, Bryn Owen, Esmeralda Parra-Peralbo, Daniel Perea and Marc Tatar for comments on the manuscript. This work was funded by grants from the Wellcome Trust, European Research Council and the Medical Research Council (WT083559, ERCStG 310411 and intramural to IM-A), Generalitat Valenciana (PROMETEO II/2013/001 to MD), Spanish Ministry of Science (BFU2009-09074, SAF2012-35181, CSD2007-00023 to MD), Botin Foundation (to MD), and the Singapore National Research Foundation (RF2010-06 to JYY). TR held a postdoctoral fellowship from the Deutsche Forschungsgemeinschaft and IM-A is a member of, and is supported by, the EMBO Young Investigator Programme.

\section{Additional information}

Funding

\begin{tabular}{|c|c|c|}
\hline Funder & Grant reference & Author \\
\hline Wellcome Trust & WT083559 & Irene Miguel-Aliaga \\
\hline European Research Council (ERC) & ERCStG 310411 & Irene Miguel-Aliaga \\
\hline Medical Research Council (MRC) & intramural & Irene Miguel-Aliaga \\
\hline $\begin{array}{l}\text { Ministerio de Ciencia e } \\
\text { Innovacion }\end{array}$ & BFU2009-09074 & Maria Dominguez \\
\hline Botin Foundation & & Maria Dominguez \\
\hline $\begin{array}{l}\text { National Research Foundation- } \\
\text { Prime Minister's office, Republic } \\
\text { of Singapore (NRF) }\end{array}$ & RF2010-06 & Joanne Y Yew \\
\hline $\begin{array}{l}\text { Generalitat Valenciana (Regional } \\
\text { Government of Valencia) }\end{array}$ & PROMETEO II/2013/001 & Maria Dominguez \\
\hline $\begin{array}{l}\text { Ministerio de Ciencia e } \\
\text { Innovacion }\end{array}$ & CSD2007-00023 & Maria Dominguez \\
\hline $\begin{array}{l}\text { Ministerio de Ciencia e } \\
\text { Innovacion }\end{array}$ & SAF2012-35181 & Maria Dominguez \\
\hline
\end{tabular}

The funders had no role in study design, data collection and interpretation, or the decision to submit the work for publication.

Author contributions

TR, JJ, Revising the article, Conception and design, Acquisition of data, Analysis and interpretation of data; PC, Writing the article, Conception and design, Acquisition of data, Analysis and interpretation of data; ZA, Conception and design, Acquisition of data; EB, KJT, Acquisition of data, Contributed unpublished essential data or reagents; JYY, Revising the article, Analysis and interpretation of data; MD, Revising the article, Conception and design, Analysis and interpretation of data; IM-A, Writing the article, Conception and design, Analysis and interpretation of data

\section{References}

Abdou MA, He Q, Wen D, Zyaan O, Wang J, Xu J, Baumann AA, Joseph J, Wilson TG, Li S, Wang J. 2011. Drosophila Met and Gce are partially redundant in transducing juvenile hormone action. Insect Biochemistry and Molecular Biology 41:938-945. doi: 10.1016/j.ibmb.2011.09.003.

Al-Anzi B, Sapin V, Waters C, Zinn K, Wyman RJ, Benzer S. 2009. Obesity-blocking neurons in Drosophila. Neuron 63:329-341. doi: 10.1016/j.neuron.2009.07.021.

Antonello ZA, Reiff T, Ballesta E, Dominguez M. 2015. Robust intestinal homeostasis relies on cellular plasticity in enteroblasts mediated by miR-8-Escargot switch. The EMBO Journal. doi: 10.15252/embj.201591517.

Athippozhy A, Huang L, Wooton-Kee CR, Zhao T, Jungsuwadee P, Stromberg AJ, Vore M. 2011. Differential gene expression in liver and small intestine from lactating rats compared to age-matched virgin controls detects increased mRNA of cholesterol biosynthetic genes. BMC Genomics 12:95. doi: 10.1186/1471-2164-12-95. 
Avila FW, Sirot LK, LaFlamme BA, Rubinstein CD, Wolfner MF. 2011. Insect seminal fluid proteins: identification and function. Annual Review of Entomology 56:21-40. doi: 10.1146/annurev-ento-120709-144823.

Bajgar A, Jindra M, Dolezel D. 2013. Autonomous regulation of the insect gut by circadian genes acting downstream of juvenile hormone signaling. Proceedings of the National Academy of Sciences of USA 110: 4416-4421. doi: 10.1073/pnas.1217060110.

Barnes Al, Wigby S, Boone JM, Partridge L, Chapman T. 2008. Feeding, fecundity and lifespan in female Drosophila melanogaster. Proceedings. Biological Sciences/The Royal Society 275:1675-1683. doi: 10.1098/ rspb.2008.0139.

Biteau B, Hochmuth CE, Jasper H. 2008. JNK activity in somatic stem cells causes loss of tissue homeostasis in the aging Drosophila gut. Cell Stem Cell 3:442-455. doi: 10.1016/j.stem.2008.07.024.

Biteau B, Karpac J, Supoyo S, Degennaro M, Lehmann R, Jasper H. 2010. Lifespan extension by preserving proliferative homeostasis in Drosophila. PLOS Genetics 6:e1001159. doi: 10.1371/journal.pgen.1001159.

Bontonou G, Shaik HA, Denis B, Wicker-Thomas C. 2015. Acp70A regulates Drosophila pheromones through juvenile hormone induction. Insect Biochemistry and Molecular Biology 56:36-49. doi: 10.1016/j.ibmb.2014.11.008.

Buchon N, Osman D, David FP, Fang HY, Boquete JP, Deplancke B, Lemaitre B. 2013. Morphological and molecular characterization of adult midgut compartmentalization in Drosophila. Cell Reports 3:1725-1738. doi: 10.1016/j.celrep.2013.04.001.

Busson D, Gans M, Komitopoulou K, Masson M. 1983. Genetic analysis of three dominant female-sterile mutations located on the X chromosome of Drosophila melanogaster. Genetics 105:309-325.

Carey HV. 1990. Seasonal changes in mucosal structure and function in ground squirrel intestine. The American Journal of Physiology 259:R385-R392.

Carvalho GB, Kapahi P, Anderson DJ, Benzer S. 2006. Allocrine modulation of feeding behavior by the Sex Peptide of Drosophila. Current Biology 16:692-696. doi: 10.1016/j.cub.2006.02.064.

Casirola DM, Ferraris RP. 2003. Role of the small intestine in postpartum weight retention in mice. The American Journal of Clinical Nutrition 78:1178-1187.

Cerf DC, Georghiou GP. 1972. Evidence of cross-resistance to a juvenile hormone analogue in some insecticideresistant houseflies. Nature 239:401-402. doi: 10.1038/239401a0.

Charles JP, Iwema T, Epa VC, Takaki K, Rynes J, Jindra M. 2011. Ligand-binding properties of a juvenile hormone receptor, Methoprene-tolerant. Proceedings of the National Academy of Sciences of USA 108:21128-21133. doi: 10.1073/pnas.1116123109.

Choi NH, Kim JG, Yang DJ, Kim YS, Yoo MA. 2008. Age-related changes in Drosophila midgut are associated with PVF2, a PDGF/VEGF-like growth factor. Aging Cell 7:318-334. doi: 10.1111/j.1474-9726.2008.00380.x.

Cognigni P, Bailey AP, Miguel-Aliaga I. 2011. Enteric neurons and systemic signals couple nutritional and reproductive status with intestinal homeostasis. Cell Metabolism 13:92-104. doi: 10.1016/j.cmet.2010.12.010.

David J, Biemont C, Fouillet P. 1974. Sur la forme des courbes de ponte de Drosophila melanogaster et leur adjustement a des modeles mathematiques. Archives de Zoologie Experimentale et Generale 115:263-267.

Economos AC, Miquel J, Binnard R, Kessler S. 1979. Quantitative analysis of mating behavior in aging male Drosophila melanogaster. Mechanisms of Ageing and Development 10:233-240. doi: 10.1016/0047-6374(79)90037-X.

Fahrbach SE, Robinson GE. 1996. Juvenile hormone, behavioral maturation, and brain structure in the honey bee. Developmental Neuroscience 18:102-114. doi: 10.1159/000111474.

Flatt T, Kawecki TJ. 2007. Juvenile hormone as a regulator of the trade-off between reproduction and life span in Drosophila melanogaster. Evolution 61:1980-1991. doi: 10.1111/j.1558-5646.2007.00151.x.

Flatt T, Moroz LL, Tatar M, Heyland A. 2006. Comparing thyroid and insect hormone signaling. Integrative and Comparative Biology 46:777-794. doi: 10.1093/icb/icl034.

Flatt T, Tu MP, Tatar M. 2005. Hormonal pleiotropy and the juvenile hormone regulation of Drosophila development and life history. BioEssays 27:999-1010. doi: 10.1002/bies.20290.

Glinoer D. 1997. The regulation of thyroid function in pregnancy: pathways of endocrine adaptation from physiology to pathology. Endocrine Reviews 18:404-433. doi: 10.1210/edrv.18.3.0300.

Gore SA, Brown DM, West DS. 2003. The role of postpartum weight retention in obesity among women: a review of the evidence. Annals of Behavioral Medicine 26:149-159. doi: 10.1207/S15324796ABM2602_07.

Gwinn ML, Lee NC, Rhodes PH, Layde PM, Rubin GL. 1990. Pregnancy, breast feeding, and oral contraceptives and the risk of epithelial ovarian cancer. Journal of Clinical Epidemiology 43:559-568. doi: 10.1016/0895-4356 (90)90160-0.

Hammond KA. 1997. Adaptation of the maternal intestine during lactation. Journal of Mammary Gland Biology and Neoplasia 2:243-252. doi: 10.1023/A:1026332304435.

Hansen M, Flatt T, Aguilaniu H. 2013. Reproduction, fat metabolism, and life span: what is the connection? Cell Metabolism 17:10-19. doi: 10.1016/j.cmet.2012.12.003.

Hildebrandt A, Bickmeyer I, Kuhnlein RP. 2011. Reliable Drosophila body fat quantification by a coupled colorimetric assay. PLOS ONE 6:e23796. doi: 10.1371/journal.pone.0023796.

Horton JD, Shah NA, Warrington JA, Anderson NN, Park SW, Brown MS, Goldstein JL. 2003. Combined analysis of oligonucleotide microarray data from transgenic and knockout mice identifies direct SREBP target genes. Proceedings of the National Academy of Sciences of USA 100:12027-12032. doi: 10.1073/pnas.1534923100.

Jiang H, Edgar BA. 2012. Intestinal stem cell function in Drosophila and mice. Current Opinion in Genetics \& Development 22:354-360. doi: 10.1016/j.gde.2012.04.002.

Jindra M, Palli SR, Riddiford LM. 2013. The juvenile hormone signaling pathway in insect development. Annual Review of Entomology 58:181-204. doi: 10.1146/annurev-ento-120811-153700. 
Jindra M, Uhlirova M, Charles J-P, Smykal V, Hill RJ. 2015. Genetic evidence for function of the bHLH-PAS protein Gce/Met as a juvenile hormone receptor. PloS Genetics (in press).

Jowett T, Postlethwait JH. 1980. The Regulation of yolk polypeptide synthesis in Drosophila ovaries and fat body by 20-hydroxyecdysone and a juvenile hormone analog. Developmental Biology 80:225-234. doi: 10.1016/00121606(80)90510-2.

Keller J, Frederking D, Layer P. 2008. The spectrum and treatment of gastrointestinal disorders during pregnancy. Nature Clinical Practice. Gastroenterology \& Hepatology 5:430-443. doi: 10.1038/ncpgasthep1197.

Klepsatel P, Galikova M, De Maio N, Ricci S, Schlotterer C, Flatt T. 2013. Reproductive and post-reproductive life history of wild-caught Drosophila melanogaster under laboratory conditions. Journal of Evolutionary Biology 26: 1508-1520. doi: 10.1111/jeb.12155.

Koren O, Goodrich JK, Cullender TC, Spor A, Laitinen K, Backhed HK, Gonzalez A, Werner JJ, Angenent LT, Knight R, Backhed F, Isolauri E, Salminen S, Ley RE. 2012. Host remodeling of the gut microbiome and metabolic changes during pregnancy. Cell 150:470-480. doi: 10.1016/j.cell.2012.07.008.

Koyama T, Mendes CC, Mirth CK. 2013. Mechanisms regulating nutrition-dependent developmental plasticity through organ-specific effects in insects. Frontiers in Physiology 4:263. doi: 10.3389/fphys.2013.00263.

Kubli E. 2003. Sex-peptides: seminal peptides of the Drosophila male. Cellular and Molecular Life Sciences 60 : 1689-1704. doi: 10.1007/s00018-003-3052.

Kunte AS, Matthews KA, Rawson RB. 2006. Fatty acid auxotrophy in Drosophila larvae lacking SREBP. Cell Metabolism 3:439-448. doi: 10.1016/j.cmet.2006.04.011.

Langevin J, Le Borgne R, Rosenfeld F, Gho M, Schweisguth F, Bellaiche Y. 2005. Lethal giant larvae controls the localization of notch-signaling regulators numb, neuralized, and Sanpodo in Drosophila sensory-organ precursor cells. Current Biology 15:955-962. doi: 10.1016/j.cub.2005.04.054.

Lee T, Luo L. 1999. Mosaic analysis with a repressible cell marker for studies of gene function in neuronal morphogenesis. Neuron 22:451-461. doi: 10.1016/S0896-6273(00)80701-1.

Lemaitre B, Miguel-Aliaga I. 2013. The digestive tract of Drosophila melanogaster. Annual Review of Genetics 47: 377-404. doi: 10.1146/annurev-genet-111212-133343.

Li M, Mead EA, Zhu J. 2011. Heterodimer of two bHLH-PAS proteins mediates juvenile hormone-induced gene expression. Proceedings of the National Academy of Sciences of USA 108:638-643. doi: 10.1073/pnas.1013914108.

Lodhi IJ, Wei X, Semenkovich CF. 2011. Lipoexpediency: de novo lipogenesis as a metabolic signal transmitter. Trends in Endocrinology and Metabolism 22:1-8. doi: 10.1016/j.tem.2010.09.002.

Marianes A, Spradling AC. 2013. Physiological and stem cell compartmentalization within the Drosophila midgut. eLife 2:e00886. doi: 10.7554/eLife.00886.

Marois E, Eaton S. 2007. RNAi in the Hedgehog signaling pathway: pFRiPE, a vector for temporally and spatially controlled RNAi in Drosophila. Methods in Molecular Biology 397:115-128. doi: 10.1007/978-1-59745-516-9_10.

McGuire SE, Le PT, Osborn AJ, Matsumoto K, Davis RL. 2003. Spatiotemporal rescue of memory dysfunction in Drosophila. Science 302:1765-1768. doi: 10.1126/science.1089035.

Micchelli CA, Perrimon N. 2006. Evidence that stem cells reside in the adult Drosophila midgut epithelium. Nature 439:475-479. doi: 10.1038/nature04371.

Middleton WR. 1971. Thyroid hormones and the gut. Gut 12:172-177. doi: 10.1136/gut.12.2.172.

Min KT, Benzer S. 1999. Preventing neurodegeneration in the Drosophila mutant bubblegum. Science 284: 1985-1988. doi: 10.1126/science.284.5422.1985.

Mirth CK, Shingleton AW. 2012. Integrating body and organ size in Drosophila: recent advances and outstanding problems. Frontiers in Endocrinology 3:49. doi: 10.3389/fendo.2012.00049.

Mirth CK, Tang HY, Makohon-Moore SC, Salhadar S, Gokhale RH, Warner RD, Koyama T, Riddiford LM, Shingleton AW. 2014. Juvenile hormone regulates body size and perturbs insulin signaling in Drosophila. Proceedings of the National Academy of Sciences of USA 111:7018-7023. doi: 10.1073/pnas.1313058111.

Moshitzky P, Fleischmann I, Chaimov N, Saudan P, Klauser S, Kubli E, Applebaum SW. 1996. Sex-peptide activates juvenile hormone biosynthesis in the Drosophila melanogaster corpus allatum. Archives of Insect Biochemistry and Physiology 32:363-374. doi: 10.1002/(SICl)1520-6327(1996)32:3/4<363::AID-ARCH9>3.0.CO;2-T.

Navare AT, Mayoral JG, Nouzova M, Noriega FG, Fernandez FM. 2010. Rapid direct analysis in real time (DART) mass spectrometric detection of juvenile hormone III and its terpene precursors. Analytical and Bioanalytical Chemistry 398:3005-3013. doi: 10.1007/s00216-010-4269-4.

Nijhout MM, Riddiford LM. 1974. The control of egg maturation by juvenile hormone in the tobacco hornworm moth, Manduca sexta. The Biological Bulletin 146:377-392. doi: 10.2307/1540412.

O'Brien LE, Soliman SS, Li X, Bilder D. 2011. Altered modes of stem cell division drive adaptive intestinal growth. Cell 147:603-614. doi: 10.1016/j.cell.2011.08.048.

Padmanabha D, Baker KD. 2014. Drosophila gains traction as a repurposed tool to investigate metabolism. Trends in Endocrinology and Metabolism 25:518-527. doi: 10.1016/j.tem.2014.03.011.

Palm W, Sampaio JL, Brankatschk M, Carvalho M, Mahmoud A, Shevchenko A, Eaton S. 2012. Lipoproteins in Drosophila melanogaster-assembly, function, and influence on tissue lipid composition. PLOS Genetics 8: e1002828. doi: 10.1371/journal.pgen.1002828.

Parker L, Gross S, Beullens M, Bollen M, Bennett D, Alphey L. 2002. Functional interaction between nuclear inhibitor of protein phosphatase type 1 (NIPP1) and protein phosphatase type 1 (PP1) in Drosophila: consequences of over-expression of NIPP1 in flies and suppression by co-expression of PP1. The Biochemical Journal 368:789-797. doi: 10.1042/BJ20020582.

Parra-Peralbo E, Culi J. 2011. Drosophila lipophorin receptors mediate the uptake of neutral lipids in oocytes and imaginal disc cells by an endocytosis-independent mechanism. PLOS Genetics 7:e1001297. doi: 10.1371/journal.pgen.1001297. 
Phillips MD, Thomas GH. 2006. Brush border spectrin is required for early endosome recycling in Drosophila. Journal of Cell Science 119:1361-1370. doi: 10.1242/jcs.02839.

Piersma T, Lindstrom A. 1997. Rapid reversible changes in organ size as a component of adaptive behaviour. Trends in Ecology \& Evolution 12:134-138. doi: 10.1016/S0169-5347(97)01003-3.

Rauschenbach IY, Karpova EK, Adonyeva NV, Andreenkova OV, Faddeeva NV, Burdina EV, Alekseev AA, Menshanov PN, Gruntenko NE. 2014. Disruption of insulin signalling affects the neuroendocrine stress reaction in Drosophila females. The Journal of Experimental Biology 217:3733-3741. doi: 10.1242/jeb.106815.

Rera M, Bahadorani S, Cho J, Koehler CL, Ulgherait M, Hur JH, Ansari WS, Lo T Jr, Jones DL, Walker DW. 2011. Modulation of longevity and tissue homeostasis by the Drosophila PGC-1 homolog. Cell Metabolism 14: 623-634. doi: 10.1016/j.cmet.2011.09.013.

Riddiford LM. 2012. How does juvenile hormone control insect metamorphosis and reproduction? General and Comparative Endocrinology 179:477-484. doi: 10.1016/j.ygcen.2012.06.001.

Roa J, Tena-Sempere M. 2014. Connecting metabolism and reproduction: roles of central energy sensors and key molecular mediators. Molecular and Cellular Endocrinology 397:4-14. doi: 10.1016/j.mce.2014.09.027.

Rogina B, Wolverton T, Bross TG, Chen K, Muller HG, Carey JR. 2007. Distinct biological epochs in the reproductive life of female Drosophila melanogaster. Mechanisms of Ageing and Development 128:477-485. doi: 10.1016/j.mad.2007.06.004.

Ryu JH, Kim SH, Lee HY, Bai JY, Nam YD, Bae JW, Lee DG, Shin SC, Ha EM, Lee WJ. 2008. Innate immune homeostasis by the homeobox gene caudal and commensal-gut mutualism in Drosophila. Science 319:777-782. doi: 10.1126/science.1149357.

Sarraf-Zadeh L, Christen S, Sauer U, Cognigni P, Miguel-Aliaga I, Stocker H, Kohler K, Hafen E. 2013. Local requirement of the Drosophila insulin binding protein imp-L2 in coordinating developmental progression with nutritional conditions. Developmental Biology 381:97-106. doi: 10.1016/j.ydbio.2013.06.008.

Schneider CA, Rasband WS, Eliceiri KW. 2012. NIH Image to ImageJ: 25 years of image analysis. Nature Methods 9:671-675. doi: 10.1038/nmeth.2089.

Seegmiller AC, Dobrosotskaya I, Goldstein JL, Ho YK, Brown MS, Rawson RB. 2002. The SREBP pathway in Drosophila: regulation by palmitate, not sterols. Developmental Cell 2:229-238. doi: 10.1016/S1534-5807(01)00119-8.

Shimano H. 2001. Sterol regulatory element-binding proteins (SREBPs): transcriptional regulators of lipid synthetic genes. Progress in Lipid Research 40:439-452. doi: 10.1016/S0163-7827(01)00010-8.

Shin SW, Zou Z, Saha TT, Raikhel AS. 2012. bHLH-PAS heterodimer of methoprene-tolerant and Cycle mediates circadian expression of juvenile hormone-induced mosquito genes. Proceedings of the National Academy of Sciences of USA 109:16576-16581. doi: 10.1073/pnas.1214209109.

Sieber MH, Thummel CS. 2012. Coordination of triacylglycerol and cholesterol homeostasis by DHR96 and the Drosophila LipA homolog magro. Cell Metabolism 15:122-127. doi: 10.1016/j.cmet.2011.11.011.

Siegmund T, Korge G. 2001. Innervation of the ring gland of Drosophila melanogaster. The Journal of Comparative Neurology 431:481-491. doi: 10.1002/1096-9861(20010319)431:4<481::AID-CNE1084>3.0.CO;2-7.

Song W, Veenstra JA, Perrimon N. 2014. Control of lipid metabolism by tachykinin in Drosophila. Cell Reports 9: 40-47. doi: 10.1016/j.celrep.2014.08.060.

Speakman JR. 2008. The physiological costs of reproduction in small mammals. Philosophical Transactions of the Royal Society of London. Series B, Biological Sciences 363:375-398. doi: 10.1098/rstb.2007.2145.

R Development Core Team. 2014. R: a language and environment for statistical Computing: R Foundation for Statistical Computing.

Theopold U, Ekengren S, Hultmark D. 1996. HLH106, a Drosophila transcription factor with similarity to the vertebrate sterol responsive element binding protein. Proceedings of the National Academy of Sciences of USA 93:1195-1199. doi: 10.1073/pnas.93.3.1195.

Tiu SH, Hult EF, Yagi KJ, Tobe SS. 2012. Farnesoic acid and methyl farnesoate production during lobster reproduction: possible functional correlation with retinoid $\mathrm{X}$ receptor expression. General and Comparative Endocrinology 175:259-269. doi: 10.1016/j.ygcen.2011.11.016.

Ueyama M, Fuyama Y. 2003. Enhanced cost of mating in female sterile mutants of Drosophila melanogaster. Genes \& Genetic Systems 78:29-36. doi: 10.1266/ggs.78.29.

Venables WN, Ripley BD. 2002. Modern applied statistics with S. New York: Springer.

Wen D, Rivera-Perez C, Abdou M, Jia Q, He Q, Liu X, Zyaan O, Xu J, Bendena WG, Tobe SS, Noriega FG, Palli SR, Wang J, Li S. 2015. Methyl farnesoate plays a dual role in regulating Drosophila metamorphosis. PLOS Genetics 11:e1005038. doi: 10.1371/journal.pgen.1005038.

Wigby S, Chapman T. 2005. Sex peptide causes mating costs in female Drosophila melanogaster. Current Biology 15:316-321. doi: 10.1016/j.cub.2005.01.051.

Wyatt GR, Davey KG. 1996. Cellular and molecular actions of juvenile hormone. II. Roles of juvenile hormone in adult insects. Advances in Insect Physiology 26:1-155. doi: 10.1016/S0065-2806(08)60030-2.

Yamamoto R, Bai H, Dolezal AG, Amdam G, Tatar M. 2013. Juvenile hormone regulation of Drosophila aging. BMC Biology 11:85. doi: 10.1186/1741-7007-11-85.

Yin C, Zou B, Jiang M, Li M, Qin W, Potter TL, Stoffolano JG. 1995. Identification of juvenile hormone III bisepoxide (JHB3), juvenile hormone III and methyl farnesoate secreted by the corpus allatum of Phormia regina (Meigen), in vitro and function of JHB3 either applied alone or as a part of a juvenoid blend. Journal of Insect Physiology 41 : 473-479. doi: 10.1016/0022-1910(94)00134-3.

Zhang Z, Xu J, Sheng Z, Sui Y, Palli SR. 2011. Steroid receptor co-activator is required for juvenile hormone signal transduction through a bHLH-PAS transcription factor, methoprene tolerant. The Journal of Biological Chemistry 286:8437-8447. doi: 10.1074/jbc.M110.191684. 